\title{
Models and Methods for Collision Analysis: A Comparison Study based on the Uber collision with a pedestrian
}

\author{
Neville A Stanton ${ }^{1,2}$, Paul M Salmon ${ }^{1,2}$, Guy H Walker ${ }^{1,2,3}$ and Maggie Stanton ${ }^{4}$ \\ ${ }^{1}$ Transportation Research Group, Department of Civil and Environmental \\ Engineering, University of Southampton, Southampton, UK
}

${ }^{2}$ Centre for Human Factors and Sociotechnical Systems, Faculty of Arts, Business and Law, University of the Sunshine Coast, Maroochydore, Queensland, Australia

${ }^{3}$ Centre for Sustainable Road Freight, Heriot-Watt University, Edinburgh, UK

${ }^{4}$ School of Psychology, Bangor University, Bangor, UK

\begin{abstract}
:
Unlike aviation, maritime and rail, road traffic collision investigation currently does not have a national investigatory body in the UK. Yet the numbers of people killed and seriously injured on the roads is far in excess of those other domains. The research presented in this paper is part of a larger project investigating the development of a road collision investigation branch in the UK. An important part of the process involves identifying a suitable accident analysis method to support investigations with the development of suitable interventions. This paper describes a study that was undertaken to identify a systemic method for investigating road collisions. Eight potential methods were identified and compared using a common incident, that of the recent Uber collision with a pedestrian. The methods were analysed against four sets of criteria: systemic, theoretical, methodological and practical. The AcciMap method, together with the accompanying Actor Map, was recommended as an appropriate approach to support road traffic collision investigations.
\end{abstract}

Keywords: Models, Methods, Road Safety, Road Traffic, Collision

\section{UK road safety compared with other transportation modes}

Whilst it is acknowledged that the UK is one of the safest countries in the world in which to travel by road, there are still around 1,700 people killed annually (DfT, 2017; this figure appears to have plateaued over the past ten years), with a total injury rate of ten times that figure. This compares unfavourably with 9 maritime fatalities in 2017 (MAIB, 2018); 85 aviation fatalities in the UK during the five-year period 2011 and 2016 (79 of these were in general aviation Eurostat, 2018), in other words upwards of 15 per year; and 309 members of the general public who died in rail related incidents in 2017 (of which 273 were suicide or suspected suicide, leaving 36 from other causes; ORR, 2017).

Additionally there was 1 rail workforce and 15 passenger fatalities (ORR, 2017).

Road crash investigation systems have previously been criticised for various limitations, including a lack of alignment with contemporary models of accident 
causation, a focus on driver behaviours, and limitations around the extent to which they can identify the full breadth of contributory factors involved (Salmon et al., 2016). Air, maritime and rail modes all have accident investigation branches, which undertake in-depth investigations with the aim, and some success, of making their transport system safer. The comparatively higher figures in road transport have led to the question of whether an accident investigation branch for roads could help to identify interventions that would reduce fatalities and serious injuries (Gooding, 2017). Indeed, it is suggested a dedicated road accident investigation branch, in addition to/support of police investigations, may lead to a cultural shift in how road accidents are viewed. Whilst a Road Collision Investigation Branch for roads in the UK could not analyse all road accidents that result in death, as there are currently 1,700 per annum, it could analyse major collisions involving multiple fatalities and indicative collisions (i.e., those accidents that keep on reoccurring). In exploring this idea it becomes important to:

- describe how accident causation models and methods have changed over time;

- provide a summary and explanation of key systemic human factors models and methods;

- give case study examples of human factors accident analysis methods; and

- provide a view on accident analysis methods together with recommendations.

Approaches to accident analysis have changed over the past century. As Figure 1 shows, the dominant theories have changed over the timeline between 1900 and 2018. In the early 1900s the focus of attention in accident investigation was the work environment, with legislation being introduced to enhance worker safety. Measures such as systematically recording accidents, providing workers with protection from equipment, breaking work into its component parts (Scientific Management: Taylor, 1911) and investigating accident-proneness (Greenwood \& Woods, 1919) led to processes that began to improve the work environment. The Domino Model as proposed by Heinrich (1931) epitomised the prevalent view of the time, wherein the aim of accident investigation was to prevent all accidents, with defences and barriers being put in place to prevent incidents from occurring. This focus continued until the 1950s, when modern day risk management began. The new approach recognised that zero risk is not achievable, rather system safety techniques benefited organisations by reducing the frequency of accidents, with the result that interest was stimulated in understanding how and why accidents occurred.

Until the 1960s the focus was largely on individual behaviour, with behaviourism as the dominant research paradigm. Dominant models at this point were Scientific Management, Accident-Proneness and the Domino Model. These models have focused on attributing blame to individuals and searching for a root cause of accidents. The associated methods based on these models (i.e. Fault Tree Analysis, Bow-Tie and STEP (Sequential Timed Event Plotting)) are linear and decompositional in nature, presenting events in a sequential manner and 
breaking them down into their component parts. As such, they tend to search for faulty components and place an emphasis on human error.

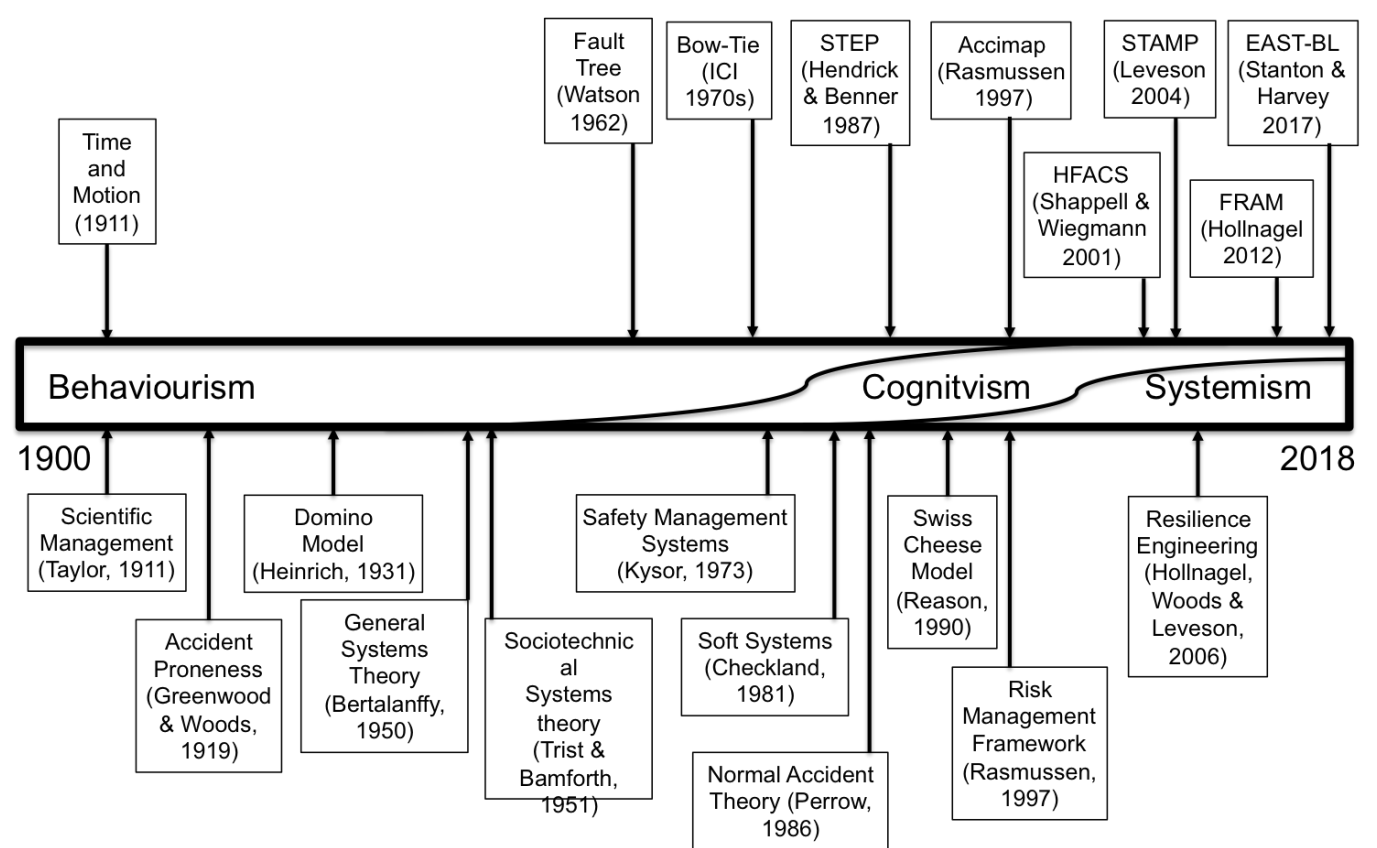

Figure 1: Timeline of selected methods (above line) and models (below line)

During the 1960s new technology and work systems required safety systems to become correspondingly more complex, necessitating structures to implement, maintain and improve them. General Systems Theory (Von Bertalanffy, 1950) proposed that all things are composed of systems and, in recognition of this, Kysor (1973) introduced the concept of a Safety Management System. A Safety Management System is used to manage or control safety. Whilst there was still a search for a root cause of any given incident, the emphasis on organisational and management features meant that accidents began to be conceptualised as having their genuine root cause in these higher level factors. Alongside this development were changes that broadened the focus still further to government and international levels, with the Health and Safety Executive being set up in the UK in 1974, increased legislation in Europe, and the World Safety Organization with its international standards being established in 1975. At the same time a number of major disasters occurred, leading to investigations and the publication of official reports, which in turn increased awareness of the multiple influences that operate when an accident happens. This led to further safety legislation and a shift from individual initiatives to a systemic approach, with large companies integrating safety management into their organisational framework. There has been an increasing recognition from that period onwards that safety management is a process rather than an outcome.

The rise of cognitivism in the 1970s led to the linking of behaviour to underlying cognitive functions and interactions with the world. This saw an increase in emphasis on psychological factors, with a focus on decision-making, particularly in relation to 'errors'. Reason (1990) made a distinction between 'errors' (unintended acts) and 'mistakes' (deliberate acts, though not malicious in 
intent). He classifies 'slips' as failures of attention, and 'lapses' as failures of memory. Both slips and lapses are examples of where the action was unintended, whereas mistakes are associated with intended action, but nevertheless having a poor outcome. A mistake occurs when an actor intentionally performs an action that turns out to be wrong. Therefore mistakes originate at the planning level, rather than the task-execution level, and can thus also be termed planning failures. This thinking has influenced the development of methods that have decisions and actions embedded within them, such as HFACS (the Human Factors Analysis and Classification Scheme) and AcciMap. The STEP method focuses on decisions and actions across time (sequentially), whilst methods such as AcciMap, HFACS (Human Factors Analysis and Classification System) and STAMP (Systems-Theoretic Accident Model and Processes) consider decisions and actions across all levels of the system.

Although Sociotechnical Systems Theory was originally developed in the 1950s, it took decades to reach mainstream risk management, and has attracted increasing interest in more recent years. It was during the 1980s that the term 'sociotechnical system' was first mentioned in relation to safety management and organisational design (Robinson, 1982). This broadened the scope of investigation to the work system (consisting of the social and technical subsystems in a given environment) as the unit of analysis. Sociotechnical Systems Theory rose in popularity from the mid-1980s with Soft Systems Methodology, Normal Accident Theory and then the Swiss Cheese Model. The Swiss Cheese Model underpins the HFACS method, viewing accidents as happening when factors align in such a way as to produce negative consequences.

Rasmussen (1997) proposed a Risk Management Framework, which highlighted the role of actors at all levels of the system as being responsible for safety. This changed the focus of accident investigation from deconstructing what had happened in terms of events, acts and errors, to designing improved systems. The approach shows that a network of interacting contributory factors underpins accidents, and that all need to be mitigated if the accident is not to reoccur in the future. The Risk Management Framework provided the theoretical basis for the AcciMap and STAMP methods (Leveson, 2004; Svedung \& Rasmussen, 2002).

Resilience engineering was developed in the mid-2000s and led to the development of FRAM (the Functional Resonance Analysis Method). Another recently proposed method is the EAST-BL (Event Analysis of Systemic Teamwork - Broken Links) technique, which is based on a general model of Sociotechnical Systems Theory and assesses resilience in task, social and information networks. Both approaches recognise that system behaviour cannot be predicted purely from the behaviour of the component parts. Each subsystem will have its own goals and functions. The models need to account for interactions between these subsystems, which may be non-linear. Alongside this there has been a shift from 'human error' to 'human performance variability'. Thus the understanding has moved from the dichotomous conceptualisation of correct and incorrect behaviour to recognising there is a range of human 
performance for which systems need to accommodate and offer resilience. Resilience methods such as FRAM and EAST-BL take a holistic approach that is not domain-specific.

It has been argued that the systemic approaches at the cutting edge of safety science in other domains are also needed to address the complexity of road safety (Salmon and Lenné, 2015). By adopting the Rasmussen (1997) Risk Management Framework, for example, it is possible to view road traffic collisions resulting from (Salmon and Lenné, 2015):

(1) multiple contributory factors rather than a single poor decision/action;

(2) the decisions and actions made by multiple system actors, rather than just road users alone;

(3) interactions between multiple contributory factors and their emergent properties (i.e., properties beyond the individual person or system component that emerge through their interaction);

(4) a lack of vertical integration across actors and events at all levels of the system;

(5) poor quality of (or absence of) communication and feedback across levels of the system, not just deficiencies at one level alone;

(6) migration of system performance over time (at multiple levels of the road transport system), from safe to unsafe, under the influence of various pressures, such as economic and physical resources and constraints; and

(7) a combination of triggering events (at multiple levels of the road transport system), each of which is unlikely in isolation to cause significant problems, but all of which occurring together can be catastrophic.

Resilience engineering (Hollnagel et al, 2006) offers a new way of thinking about the dynamics of a system. More resilient systems have a greater ability to return to a stable state after some disturbance. In road transport terms, this means the ability of the road system to either prevent collisions or to return quickly to normal running after a collision has occurred. At a broader systems level resilience means that a road transport system could respond to a set of recurring incidents (e.g. distraction-related crashes). Other studies comparing accident investigation methods have been conducted, but typically the comparisons only involve two to three methods (Salmon et al, 2012; Stanton et al, 2012;

Underwood and Waterson, 2014). In addition, none of these studies has focused on road collisions, the domains being led-outdoor activities (Salmon et al, 2012), defence (Stanton et al, 2012) and rail (Underwood and Waterson, 2014) respectively. There have been studies of road freight crashes (Newnam and Goode, 2015; Newman et al, 2017) but these are very specialized as they focused on professional drivers who drive comparatively long distances in Heavy Good 
Vehicles (HGV). Nevertheless, the work developed classification schemes for the contributory factors at the six levels of the AcciMap. The classification schemes are extremely useful for aggregating data from many collisions as well as guiding the analyst in the development of an AcciMap. The aim of the present study was to assess the applicability of the methods for investigation of road collisions, with a particular focus on light (i.e., not HGV) automated vehicles.

\section{Methods Selected for Review}

Eight accident analysis methods were selected for the current review on the basis of popularity in the literature (AcciMap, Fault Tree Analysis (FTA), HFACS and STAMP-CAST), reading of the contemporary literature on sociotechnical systems methods (EAST-BL and FRAM) and speaking with accident analysis and investigation subject matter experts (Bow-Tie and STEP), as shown in Table 1. The number of citations to each of the methods from three different databases is shown (undertaken 18 October 2018). The last row shows the number of citations against method and the specific domain of "Road Traffic Accident" (RTA).

Table 1: List of methods and citations from three different databases (*EAST (not BL) **not to do with the Bow-Tie risk method)

\begin{tabular}{|c|c|c|c|c|c|c|c|c|}
\hline Domain & AcciMap & Bow-Tie & EAST-BL & $\begin{array}{l}\text { Fault } \\
\text { Tree }\end{array}$ & FRAM & HFACS & STAMP & STEP \\
\hline $\begin{array}{l}\text { Web of } \\
\text { Science }\end{array}$ & 54 & 224 & $1\left(16^{*}\right)$ & 2,422 & 54 & 160 & 52 & 4 \\
\hline Scopus & 56 & 349 & $1\left(20^{*}\right)$ & 5,682 & 74 & 223 & 93 & 5 \\
\hline $\begin{array}{l}\text { Google } \\
\text { Scholar }\end{array}$ & 632 & 15,900 & $\begin{array}{l}42 \\
\left(270^{*}\right)\end{array}$ & 53,100 & 843 & 2,930 & 79 & 104 \\
\hline $\begin{array}{l}\text { Method } \\
+ \text { RTA }\end{array}$ & 5 & $4\left(41^{* *}\right)$ & 4 & 91 & 3 & 28 & 2 & 1 \\
\hline Notes: & \multicolumn{8}{|c|}{$\begin{array}{l}\text { EAST-BL: Event Analysis of Systemic Teamwork - Broken Links; } \\
\text { FTA: Fault Tree Analysis; } \\
\text { FRAM: Functional Resonance Analysis Method; } \\
\text { HFACS: Human Factors Analysis and Classification Scheme; } \\
\text { STAMP-CAST: Systems-Theoretic Accident Model and Processes - Causal Analysis } \\
\quad \text { using Systems Theory; } \\
\text { STEP: Sequential Timed Event Plotting }\end{array}$} \\
\hline
\end{tabular}

By far the most cited method is Fault Tree Analysis, however it has also been established as an accident analysis and investigation method the longest, as shown in table 2 .

The method, associated model type, creator(s), date and source are presented in table 2. The literature is vague on the source of the Bow-Tie model, suggesting that Imperial Chemistry Industries (ICI) developed it sometime in the 1970s. The literature is similarly vague on the source of FTA, suggesting that it was 
developed in Bell Laboratories in 1962. Sources of all the other method are provided in the references.

Table 2: List of methods and corresponding models

\begin{tabular}{|c|c|c|c|c|}
\hline Method & Model type & Creator(s) & Date & Source \\
\hline AcciMap & Heterarchy & Rasmussen & 1997 & Safety Science \\
\hline Bow-Tie & $\begin{array}{l}\text { Tree } \\
\text { structure }\end{array}$ & ICI? & 1970s? & ICI? \\
\hline EAST-BL & Networks & $\begin{array}{l}\text { Stanton and } \\
\text { Harvey }\end{array}$ & 2017 & Ergonomics \\
\hline FTA & $\begin{array}{l}\text { Tree } \\
\text { structure }\end{array}$ & Watson & 1962 & Bell Laboratories \\
\hline FRAM & Network & Hollnagel & 2012 & Book (Ashgate) \\
\hline HFACS & Taxonomic & $\begin{array}{l}\text { Shappell and } \\
\text { Wiegmann }\end{array}$ & 2001 & $\begin{array}{l}\text { Human Factors and } \\
\text { Aerospace Safety }\end{array}$ \\
\hline STAMP-CAST & $\begin{array}{l}\text { Control } \\
\text { structure }\end{array}$ & Leveson & 2004 & Safety Science \\
\hline STEP & Multilinear & $\begin{array}{l}\text { Hendrick and } \\
\text { Benner }\end{array}$ & 1987 & $\begin{array}{l}\text { Book (Marcel } \\
\text { Dekker) }\end{array}$ \\
\hline \multicolumn{5}{|r|}{ Inks; } \\
\hline
\end{tabular}

The source reference for each of the methods in table 2 is contained within the reference section of this paper. A short description of each of the methods is contained within Appendix A. The application the methods to a case study may be found in Appendix B.

\section{Comparison of Methods}

A comparison of the methods was undertaken across a range of systemic (criteria derived from the six systems levels: Rasmussen, 1997), theoretical (criteria derived from the seven tenets of accidents, Rasmussen, 1997), methodological (criteria derived from the study of methods, Stanton et al, 2013, 2014) and practical (criteria derived from the usability and usefulness, Stanton et al, 2013,2014) criteria as shown in tables 3 to 6. 
As Table 3 shows, the systems levels are represented on the vertical axis and the eight methods in alphabetical order on the horizontal axis. It is important that the accident analysis methods are able to address all of the levels in the system, as systemic approaches are most likely to identify underlying, multiple, interacting causes of accidents. Whilst any analyst could subjectively include any level in their analysis, the comparison was based on what is typically analysed and what the methods explicitly guide. The AcciMap and STAMP-CAST methods (highlighted in red) address all of the levels in the system, from equipment and environment at the lowest level all the way up to government policy and budgeting at the highest level. This means that these two methods are recommended from a system coverage standpoint.

\section{Table 3: Evaluation of methods against the system levels}

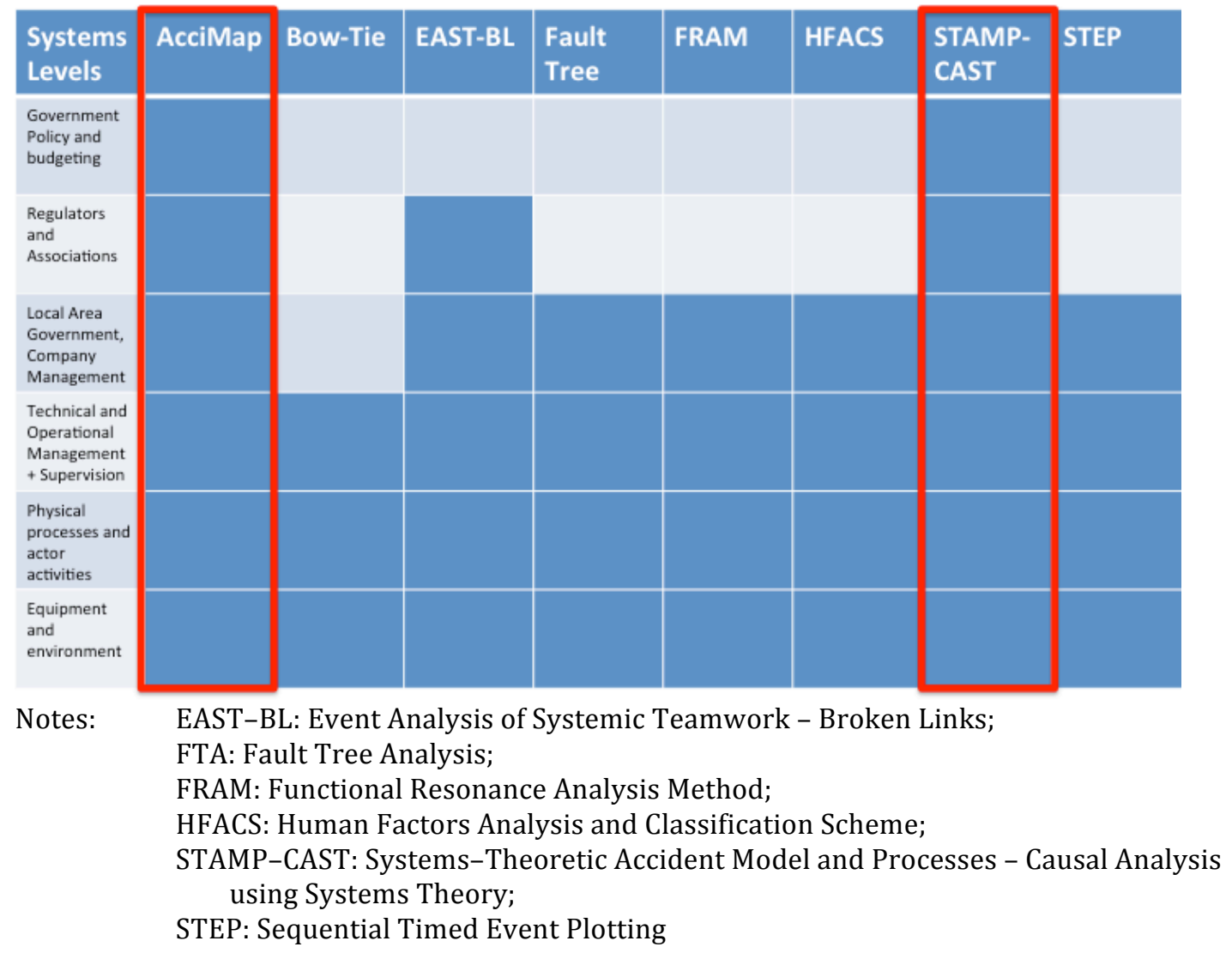

Table 4 shows an evaluation of the methods against Rasmussen's seven tenets of accidents of accident causation as outlined earlier. It is widely acknowledged that these tenets are the state-of-the-art principles of accident causation identified in the scientific literature (Rasmussen, 1997). It is important that an accident analysis method is able to account for each of these tenets in its representation. No method covered all seven tenets, and only the AcciMap method (highlighted in red) covered six of the seven of the tenets (missing the migration of performance from safe to unsafe). To undertake the latter would require the method to have a dynamic aspect that could animate performance migration. Perhaps it is no surprise that AcciMaps perform well against these criteria as they are based on the original work from Rasmussen (1997), which 
underpins the AcciMap method. Nevertheless, AcciMap is recommended from an accident tenets standpoint.

Table 4: Evaluation of methods against the seven tenets of accidents

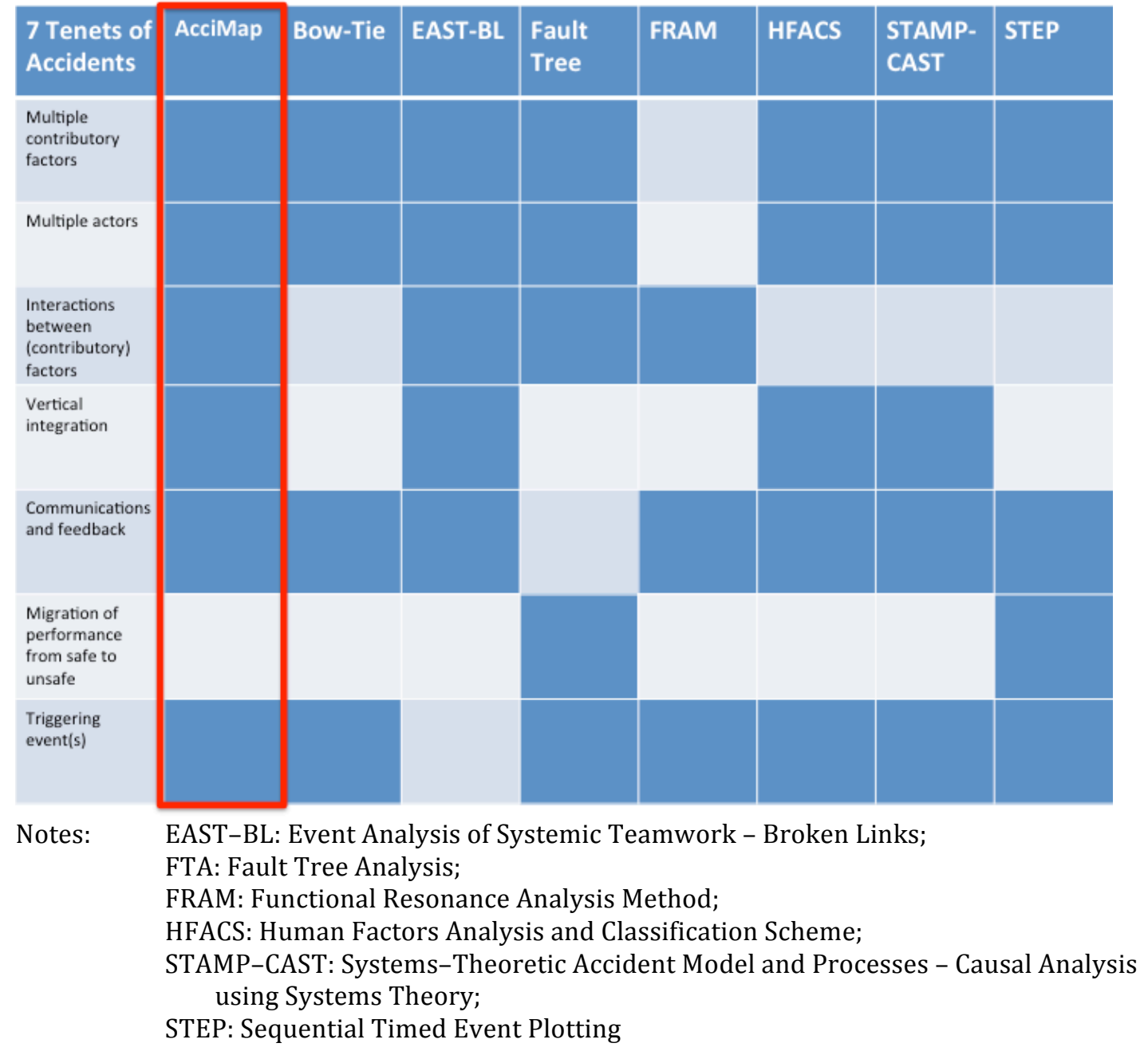

An evaluation of the eight methods against seven methodological criteria (as shown in the vertical axis of Table 5) was also undertaken. The AcciMap and fault tree methods (highlighted in red) were rated as having more methodological integrity than the other six methods. The lack of an inbuilt classification scheme is judged to be a weakness in both of these methods. That is, whilst approaches such as HFACS provide a system to support the identification and classification of contributory factors, Accimap and fault tree methods do not. Whilst this enhances the methods flexibility, it raises concerns regarding reliability when used by different analysts. Nevertheless, the AcciMap and Fault Tree methods are recommended from a methodological standpoint. Notably, classification schemes have been developed to support the use of AcciMap in other areas such as led outdoor recreation (Salmon et al., 2017) and HGV crashes (Newnam and Goode, 2015; Newman et al, 2017). 
Table 5: Evaluation of methods against the methodological criteria

\begin{tabular}{|c|c|c|c|c|c|c|c|c|}
\hline Criteria & AcciMap & Bow-Tie & EAST-BL & $\begin{array}{l}\text { Fault } \\
\text { Tree }\end{array}$ & FRAM & HFACS & $\begin{array}{l}\text { STAMP- } \\
\text { CAST }\end{array}$ & STEP \\
\hline $\begin{array}{l}\text { Evidence of } \\
\text { reliability }\end{array}$ & Medium & N/A & N/A & High & N/A & Medium & N/A & N/A \\
\hline $\begin{array}{l}\text { Evidence of } \\
\text { validity }\end{array}$ & Medium & N/A & N/A & High & N/A & Medium & N/A & N/A \\
\hline $\begin{array}{l}\text { Complexity } \\
\text { of approach }\end{array}$ & Low & Medium & Medium & Medium & High & Low & High & Low \\
\hline $\begin{array}{l}\text { Reliance on } \\
\text { subject } \\
\text { matter } \\
\text { experts }\end{array}$ & Medium & Medium & High & High & Medium & Medium & High & Medium \\
\hline $\begin{array}{l}\text { Auditability } \\
\text { and } \\
\text { traceability } \\
\text { of system } \\
\text { influences }\end{array}$ & High & Medium & Medium & Medium & Medium & Medium & Medium & Medium \\
\hline $\begin{array}{l}\text { Applicability } \\
\text { to analysis of } \\
\text { road traffic } \\
\text { accidents }\end{array}$ & High & Medium & Low & Medium & Low & Medium & High & High \\
\hline $\begin{array}{l}\text { In-built } \\
\text { classification } \\
\text { scheme of } \\
\text { contributory } \\
\text { factors }\end{array}$ & No & No & No & No & No & Yes & Yes & No \\
\hline $\begin{array}{ll}\text { otes: } & \text { E } \\
& \text { F } \\
F^{\prime} \\
\mathrm{F} 1 \\
\mathrm{H} \\
\mathrm{S}\end{array}$ & \multicolumn{8}{|c|}{$\begin{array}{l}\text { N/A = not applicable } \\
\text { EAST-BL: Event Analysis of Systemic Teamwork - Broken Links; } \\
\text { FTA: Fault Tree Analysis; } \\
\text { FRAM: Functional Resonance Analysis Method; } \\
\text { HFACS: Human Factors Analysis and Classification Scheme; } \\
\text { STAMP-CAST: Systems-Theoretic Accident Model and Processes - Causal Analysis } \\
\quad \text { using Systems Theory; }\end{array}$} \\
\hline
\end{tabular}

Finally, an evaluation of the eight methods against six practical criteria (see the vertical axis of Table 6) was undertaken. These include usability criteria and evidence of practical impact. The practical criteria were assessed by the authors of the paper based in their expertise, knowledge of the field and having undertaken the analysis of the collision using the methods for themselves. The AcciMap method (highlighted in red) was rated as more practical than the other seven methods. 
Table 6: Evaluation of methods against the practical criteria

\begin{tabular}{|c|c|c|c|c|c|c|c|c|}
\hline Criteria & AcciMap & Bow-Tie & EAST-BL & $\begin{array}{l}\text { Fault } \\
\text { Tree }\end{array}$ & FRAM & HFACS & $\begin{array}{l}\text { STAMP- } \\
\text { CAST }\end{array}$ & STEP \\
\hline $\begin{array}{l}\text { Ease of use } \\
\text { (High=easy) }\end{array}$ & High & Medium & Medium & Medium & Low & High & Low & High \\
\hline $\begin{array}{l}\text { Application } \\
\text { time } \\
\text { (Low=quick) }\end{array}$ & Low & Medium & Medium & Medium & High & Medium & High & Medium \\
\hline $\begin{array}{l}\text { Training } \\
\text { demand } \\
\text { (Low=little } \\
\text { training } \\
\text { demand) }\end{array}$ & Low & Medium & High & Medium & High & Low & Medium & Low \\
\hline $\begin{array}{l}\text { Simplicity of } \\
\text { interpretation } \\
\text { (High=simple) }\end{array}$ & High & High & Medium & High & Low & High & Medium & High \\
\hline $\begin{array}{l}\text { Tools required } \\
\text { (Low=no tools } \\
\text { above pen and } \\
\text { paper) }\end{array}$ & Low & Low & Low & Low & Medium & Medium & Medium & Low \\
\hline $\begin{array}{l}\text { Evidence of } \\
\text { practical impact } \\
\text { (High=good) }\end{array}$ & Medium & High & Low & High & Medium & Medium & Medium & Medium \\
\hline Notes: & \multicolumn{8}{|c|}{$\begin{array}{l}\text { EAST-BL: Event Analysis of Systemic Teamwork - Broken Links; } \\
\text { FTA: Fault Tree Analysis; } \\
\text { FRAM: Functional Resonance Analysis Method; } \\
\text { HFACS: Human Factors Analysis and Classification Scheme; } \\
\text { STAMP-CAST: Systems-Theoretic Accident Model and Processes - Causal Analysis } \\
\quad \text { using Systems Theory; } \\
\text { STEP: Sequential Timed Event Plotting }\end{array}$} \\
\hline
\end{tabular}

In summary, the AcciMap method was evaluated as performing best across all of the theoretical, methodological and practical criteria. As such, it is the recommended approach for use in road accident investigation studies. This is in line with other studies that have recommended a similar approach in road transport (Newnam et al., 2017; Salmon et al., 2012). An example of application of the Actor Map and AcciMap methods applied to a road collision is presented in the next section.

\section{Case Study Based on the Uber Vehicle Collision with a Pedestrian}

This case study was based on the Uber vehicle collision with a pedestrian wheeling a bicycle across the road. The decision to analyse the Uber collision was based on the desire that any chosen method should be able to analyse collision for both manual and automated vehicles. The collision between the Uber vehicle and the pedestrian was very similar to conventional vehicles collisions with pedestrians and cyclists (vulnerable road users). The accident occurred at approximately 21.58 on 18th March 2018 in Arizona, USA. Although the full report by NHTSA (the US National Highway Traffic Safety Administration) was not available at the time of this study, there was sufficient information available to undertake analysis with the methods selected for review (a short preliminary report was available: NTSB, 2018) together with material from online news websites indicated at the bottom of table 7 . The timeline of the immediate events leading up to the collision, as far as they are known, are presented in Table 7. 
Table 7: Timeline of events leading to Uber vehicle collision with pedestrian

\begin{tabular}{|ll|}
\hline Time & Event \\
\hline 21.14 & $\begin{array}{l}\text { 44-year-old Rafaela Vasquez arrives for work at the Uber facilities in } \\
\text { Tempe, Arizona. }\end{array}$ \\
& $\begin{array}{l}\text { Vasquez leaves the Tempe facilities in a self-driving } 2017 \text { Volvo } \\
\text { XC90 operated by Uber to run an established test route through } \\
\text { downtown Tempe. }\end{array}$ \\
\hline 21.39 & The vehicle is switched to autonomous mode. \\
\hline Unknown & $\begin{array}{l}\text { A report from Tempe police (contested by Vasquez) alleges that } \\
\text { Vasquez began streaming The Voice on the Hulu app on a } \\
\text { smartphone. During this time the Tempe police allege that Vasquez } \\
\text { can be seen frequently looking down at the lower centre console } \\
\text { area near her knee and frequently smirking and laughing. Her hands } \\
\text { were not visible in the frame of the surveillance footage. Police } \\
\text { determine she looks down } 204 \text { times over the course of } 11.8 \text { miles. } \\
\text { Her eyes were off of the road for } 6 \text { min } 47 \text { sec during this period [i.e. } \\
\text { over 25\% of time]. }\end{array}$ \\
\hline 21.58 & $\begin{array}{l}\text { Vasquez looks up while driving northbound on Mill Avenue toward } \\
\text { Curry Road, approximately 0.5 seconds before the crash. She } \\
\text { attempts to swerve left before striking 49-year-old Elaine Herzberg } \\
\text { at 39 mph [speed zone posted at } 45 \text { mph] as she crosses the street } \\
\text { mid-block. Hulu's records also show the streaming of the show } \\
\text { ended at this time. }\end{array}$ \\
\hline $\begin{array}{l}\text { Vasquez calls } 911 \text { and is released later that night after speaking to } \\
\text { police. She stated she was monitoring the self-driving system } \\
\text { interface and neither her business nor personal phones were in use. }\end{array}$ \\
(Approx.)
\end{tabular}

Sources: https://eu.azcentral.com/story/news/local/tempe/2018/06/22/fatal-uber-crashtimeline-crash-and-investigation/725921002/ and

https://www.ntsb.gov/investigations/AccidentReports/Reports/HWY18MH010-prelim.pdf

The paths taken by the pedestrian and the vehicle are indicated in figure 2 . 


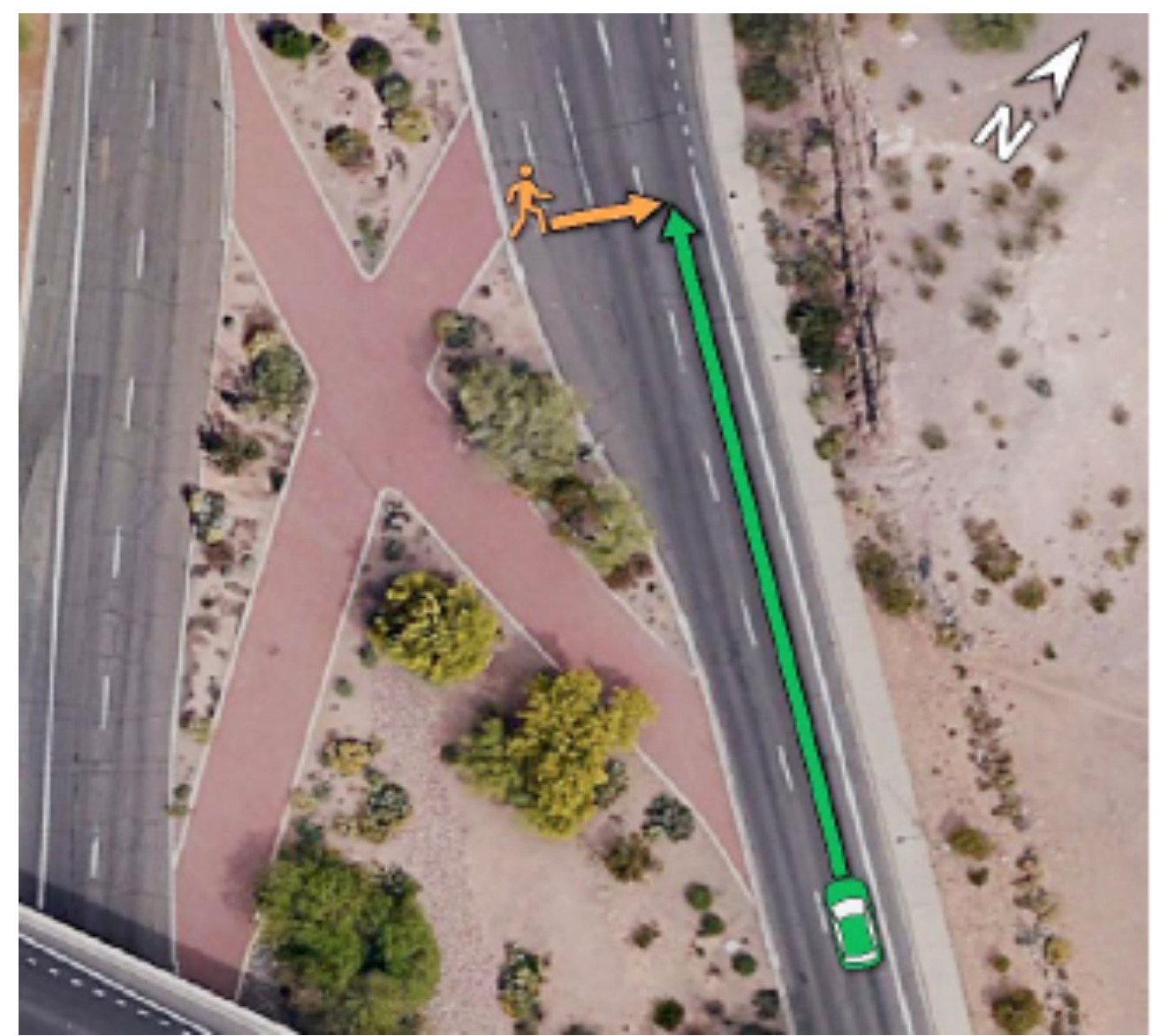

Figure 2: Paths of the pedestrian and vehicle leading to the collision

\section{Background}

Uber was originally planning to undertake its testing in California but there was a dispute over the need for permits to operate the automated vehicle. Uber argued that as a driver was present, no permit was necessary, but the California regulators disagreed and revoked the Uber vehicle registrations. On hearing this, the governor of Arizona encouraged Uber to continue its testing in his state. This decision was likely based on the perceived economic growth expected to follow investment in the development of autonomous vehicles. Uber set up its testing programme in Arizona with plans to conduct on-road studies. There is considerable competition between companies to have the first on-road fully autonomous vehicle.

With regard to the test-vehicle and its functioning, a decision was taken by the Uber engineers to disable the Volvo City Safety system (including the AEB system) as it can induce an erratic ride experience if false obstructions are detected. Uber recruited drivers who were trained over three weeks to operate the vehicle. They were to work eight-hour shifts, monitoring the vehicle as it drove around a preset route. The monitoring task involved observing the automated vehicle's functioning and noting any abnormalities or points of interest on a tablet mounted in the centre console. The vehicle operators were also supposed to regain control of the vehicle in the event of an emergency. Following the collision of the Uber vehicle with the pedestrian, the testing 
programme was suspended. There is an ongoing investigation into the collision (NTSB, 2018).

\section{Analysis of the Accident}

Analysis of the accident was undertaken using all eight methods in order to highlight the differences between the approaches. The AcciMap analysis is presented next, and the other seven analyses are presented in Appendix B.

The AcciMap process begins with an Actor Map to identify the main stakeholders who share the responsibility for safety in the road transport system in which the collision occurred. The Actor Map is shown in Figure 2. This shows eight levels of the system, from 'equipment and environment' at the lowest level up to 'international influences', the highest level. The next step is to identify the contribution (or lack of contribution) of each actor that influenced the events leading up to the collision, as shown in the AcciMap in Figure 3.

\begin{tabular}{|c|c|c|c|c|c|}
\hline $\begin{array}{l}\text { Interemational } \\
\text { inffunences }\end{array}$ & 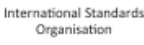 & & & & \\
\hline $\begin{array}{c}\text { National } \\
\text { cominites }\end{array}$ & 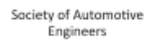 & & & & \\
\hline $\begin{array}{l}\text { Federal and State } \\
\text { Goverment }\end{array}$ & rederal Government & 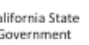 & $\begin{array}{l}\text { Arizon State } \\
\text { Governmente }\end{array}$ & & \\
\hline 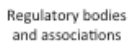 & 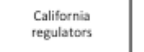 & 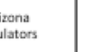 & & & \\
\hline 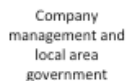 & Uber & ivo & $\begin{array}{l}\text { cutan } \\
\text { planners }\end{array}$ & & \\
\hline $\begin{array}{l}\text { Technical and } \\
\text { operatonan } \\
\text { management }\end{array}$ & Uber enginineers & & & & \\
\hline Driving processes & Defiver & & & & \\
\hline 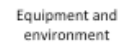 & $\begin{array}{l}\text { Autumated } \\
\text { vethice }\end{array}$ & Mestion & unction & Bigycte & Signage \\
\hline
\end{tabular}

Figure 3: Actor Map of the Uber collision with a pedestrian

The events, failures, decisions and actions that interacted to create the Uber collision are presented in the boxes in Figure 4, with the interactions between them indicated by the arrows. At the top of the AcciMap, the lack of international and national standards for automation design and testing meant that Uber had no technical guidance for appropriate interfaces, safety standards, or testing regimes. The task of the Uber test driver required them to look at the road scene, evaluate the performance of the vehicle, and make notes as required on the tablet. As already noted in Table 3, the driver looked up approximately half a second before the collision and, on spotting the pedestrian wheeling a bicycle across the road (taking a direct route to a homeless shelter), she grabbed the steering to attempt a swerve. Although the vehicle automation had identified the pedestrian (on its third attempt) and activated the AEB, it did not respond because the Uber engineers had disabled it. The pedestrian was struck at a speed 
of $39 \mathrm{mph}$ and died in a local hospital. It was also noted that the pedestrian was not crossing the road at the pedestrian crossing. Although the crossing had the appearance of a pedestrian crossing, there were small, unlit, signs stating that the real crossing was further up the road. It is possible that the pedestrian may not have seen the signs (as there was no roadway lighting). It is equally possible that a strong 'desire line' existed in this location, which would render the appeals from signs ineffective in any case. The autopsy revealed that the pedestrian was intoxicated with methamphetamine and marijuana.

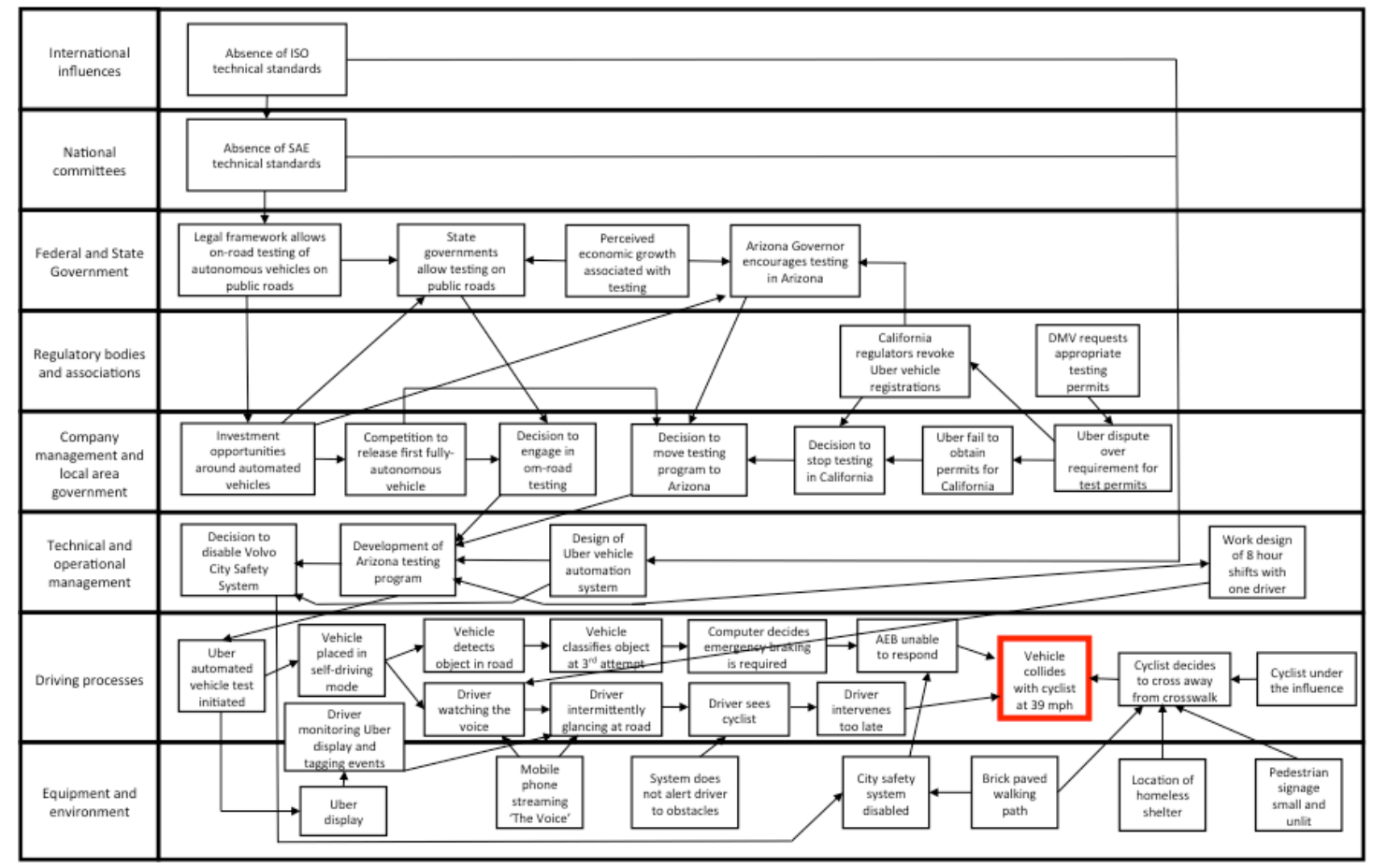

Figure 4: AcciMap of the Uber collision with a pedestrian

Sources: As table 7 and https://www.theguardian.com/technology/2018/mar/28/uber-arizonasecret-self-driving-program-governor-doug-ducey and https://www.bbc.com/news/technology-44243118 and https://www.citylab.com/transportation/2018/03/former-uber-backup-driver-we-saw-thiscoming/556427/

From Accimap, it is possible to develop recommendations with the aim of preventing this type of event from reoccurring. Examples of the type of recommendations that could be developed are illustrated in Table 4. At the top two levels (international influences and national committees), new standards for vehicle automation and on-road testing are required. Governments and regulatory bodies (the next two levels down) need to develop and enforce new laws for vehicle automation and their on-road testing. At the next level down, the company needs to undertake a comprehensive analysis of human and technical risks, accompanied by task and workload analysis. At the same level, local planners should improve lighting and fence off central reservations where there is a natural crossing point. Technical and operational management need to better understand the demands made on drivers of automated vehicles and share tasks accordingly. The test vehicles should be fitted with dual control and probably have two drivers present. The inbuilt vehicle safety systems should be left intact. The pedestrian, too, should cross at the designated point and avoid impairment 
due to drugs. Finally, at the bottom level, drivers should place all nomadic devices in the glovebox before the vehicle is put in motion. The point here is that collisions do not result from any single point of failure; rather they are systemic and multicausal in nature. To reduce collisions, issues need to be addressed at all of the system levels. This means that any method for road collision investigation needs to take a systemic approach before reductions in the 1,700 deaths on UK roads are likely to occur. 
Table 8: List of potential recommendations

\begin{tabular}{|c|c|}
\hline System levels & Potential recommendations \\
\hline \multirow[t]{2}{*}{$\begin{array}{l}\text { International } \\
\text { influences }\end{array}$} & $\begin{array}{l}\text { Develop new standards for vehicle automation (e.g. head-up } \\
\text { interface) }\end{array}$ \\
\hline & $\begin{array}{l}\text { Develop new standards for on-road testing of vehicle } \\
\text { automation (e.g. two testers in vehicle) }\end{array}$ \\
\hline \multirow{2}{*}{$\begin{array}{l}\text { National } \\
\text { committees }\end{array}$} & Develop new standards for vehicle automation \\
\hline & $\begin{array}{l}\text { Develop new standards for on-road testing of vehicle } \\
\text { automation }\end{array}$ \\
\hline \multirow{3}{*}{$\begin{array}{l}\text { Federal and } \\
\text { state } \\
\text { government }\end{array}$} & Develop new laws on vehicle automation \\
\hline & Develop new laws for on-road testing of vehicle automation \\
\hline & Require permits for on-road testing of vehicle automation \\
\hline \multirow{3}{*}{$\begin{array}{l}\text { Regulatory } \\
\text { bodies and } \\
\text { associations }\end{array}$} & Enforce new laws on vehicle automation \\
\hline & Enforce new laws for on-road testing of vehicle automation \\
\hline & Enforce permits for on-road testing of vehicle automation \\
\hline \multirow{5}{*}{$\begin{array}{l}\text { Company } \\
\text { management } \\
\text { and local area } \\
\text { government }\end{array}$} & Uber: Undertake comprehensive driver task analysis \\
\hline & Undertake comprehensive analysis of human and technical risks \\
\hline & Analyse the workload of human driver with automation \\
\hline & $\begin{array}{l}\text { City Planners: Fence off central reservations that are not part of } \\
\text { pedestrian crossings }\end{array}$ \\
\hline & Improve highway lighting \\
\hline \multirow{4}{*}{$\begin{array}{l}\text { Technical and } \\
\text { operational } \\
\text { management }\end{array}$} & $\begin{array}{l}\text { Conduct pilot studies with human drivers to discover potential } \\
\text { problems }\end{array}$ \\
\hline & $\begin{array}{l}\text { Share tasks between two drivers to ensure sufficient rests (eyes- } \\
\text { out versus eyes-in tasks) and swap tasks regularly }\end{array}$ \\
\hline & Leave safety systems intact (including the AEB) \\
\hline & $\begin{array}{l}\text { Fit dual controls to vehicle so that both drivers can drive the } \\
\text { vehicle manually if required }\end{array}$ \\
\hline $\begin{array}{l}\text { Driving } \\
\text { processes }\end{array}$ & $\begin{array}{l}\text { Ensure that one driver is eyes-out at all times and swap tasks } \\
\text { between drivers regularly }\end{array}$ \\
\hline $\begin{array}{l}\text { Equipment and } \\
\text { environment }\end{array}$ & $\begin{array}{l}\text { Place all nomadic devices (such as phones) in glovebox before } \\
\text { the vehicle is driven }\end{array}$ \\
\hline
\end{tabular}

The case studies applying the methods to the Uber collision are contained within Appendix B. 


\section{Discussion}

The learnings and safety impacts of a national accident investigation body are highly dependent on the method that they use to investigate incidents. In this study we compared and contrasted 8 methods to identify which was most suited for use as part of a national road crash investigation body in the UK. Based on a comparison and evaluation against three sets of criteria (Tables 3 to 6), the AcciMap method was found to be the most suitable out of the eight methods assessed.. There were trade-offs, however, as STAMP-CAST addressed all of the system levels (Table 3) and Fault Tree Analysis performed equally well on the methodological criteria (Table 5). Overall, though, Accimap performed the best against these specific criteria. This confirms the findings from previous studies in different domains (Salmon et al, 2012; Stanton et al, 2012; Underwood and Waterson, 2014; Newnam and Goode, 2015; Newman et al, 2017). Other methods comparison studies have also found AcciMap to be superior (Ledoutdoor education, defence, rail applications and road freight) so this finding does appear to generalise across different domains. The culmination of evidence seems to suggest that AcciMap and the associated Actor Map method could be domain independent and a good match to the road accident investigation domain.

Various scholars have criticised road crash investigation systems for not identifying contributory factors beyond driver, the vehicle, and the road environment (Larsson et al., 2010; Newnam et al., 2017; Salmon et al., 2012; 2016). Very few of the methods addressed all of the system levels from equipment to the environment to governmental policy and budgeting (apart from AcciMap and STAMP-CAST). Most of the remaining methods only addressed four of the six levels with the exceptions of EAST-BL (five levels) and Bow-Tie (three levels). A truly systemic approach needs to be able to address all of the levels to understand the contributory factors and develop systems-based recommendations. Recent developments in the Actor Map and AcciMap method have added two further levels: national committees and international committees (Parnell et al, 2017). There is no reason why road collision investigation analysis could not be scaled up to all eight levels, as was undertaken in the case study. Indeed, whether further work could explore the efficacy of existing levels and whether new ones should be developed.

In terms of Rasmussen's (1997) seven tenets of accident causation, AcciMap addressed six, but not the migration of performance from safe to unsafe tenet. Most of the other methods were found to address 5 tenets, except Bow-Tie which addressed 4 and FRAM only 3. Clearly AcciMap performed best on this set of criteria, but would require dynamic modelling to deal with the migration of performance tenet. Causal Loop Diagrams (CLDs) and systems dynamics are methods that could be integrated with AcciMap to provide this capacity. CLDs is a method that is used to elicit and represent the interacting positive and negative feedback loops that influence system behaviour (Sterman, 2000). These could be used to show how a system migrated from a safe to unsafe state. Indeed, some 
applications of the STAMP-CAST method use CLDs for this purpose (Leveson, 2004).

With respect to the methodological criteria, most of the methods have scant, if any, information about reliability and validity. Arguably, these are the most important methodological criteria. Proof about how well a method performs on successive occasions and with alternative analysts (reliability) and it how well it detects underlying causal influences (validity) seems essential for any collision investigation method (Stanton, 2016). All Human Factors and Ergonomics methods should have to demonstrate they have met the criteria for both reliability and validity as proposed by Stanton and Young (1999a,b). Accident investigators would be well served by exploring the benefits of other methods rather than relying upon the approach they always use (Stanton and Young, 1998). Many of the methods are, in fact, simpler than they first appear (Stanton and Young, 2003). On the remaining methodological criteria, complexity, subject matter experts, auditability and applicability to road traffic collisions, both AcciMap and FTA perform acceptably (taken together with their performance on reliability and validity). Two of the methods have formal, in-built, classification systems (HFACS and STAMP), which can be useful in providing structure for the analyst (Salmon et al., 2012; Stanton and Young, 2003; Stanton et al, 2013).

The comparison of the eight methods against the practical criteria showed the AcciMap to be more efficacious than the other seven methods. It was rated well against ease of use, application time, training demand, simplicity of interpretation and tools required. These criteria have been used in other comparisons of methods to enable analysts to select from alternate methods (Stanton et al, 2013; Stanton et al, 2014). There is almost no investigation of light vehicle fleets from a pure systems thinking perspective (Talbot et al, 2013; Warmerdam et al, 2017). A system-based approach to investigations in light vehicles could help to understand the systemic causes and implementations of the findings and recommendations could help to reduce collisions in the future.

As far as the analysis of the collision between the Uber vehicle and the pedestrian pushing the bicycle is concerned, the Actor Map identified 19 actors that could have had some influence on events. At present, selection of possible actors is at the discretion of the analyst. This process could be assisted by the development of an in-built actor classification system. Such an approach has been developed by McIlroy et al (2018), who considered all possible actors for road traffic collisions in five different countries as part of a global road safety project: Bangladesh, China, Kenya, UK and Vietnam. The actor map guides the identification of decisions, actions and events in the AcciMap, where the analyst is required to consider how each actor contributed to the collision either by their activities or failure to act. The AcciMap shows 39 such decisions, actions and events leading up to the collision. Studies into road freight crashes have identified generic contributory factors at all system levels (Newman and Goode, 2015; Newman et al, 2017), some of which were present in this case study, such as inadequate regulations, work schedule, in-vehicle distraction, alcohol and drugs. For example, at the governmental level, the absence of technical standards has led to inadequate regulation of the automated vehicles. At the 
operational management level, the work schedule for vehicle operators seemed inappropriate (i.e., working along on eight hour shifts). At the driving process level, these were in-vehicle distractions (such as attending to the Uber display). Also at the driving process is the issue of the pedestrian/cyclist being under the influence of drugs. Whilst the classification system developed by Newman (2015) and extended by Newman et al (2017) is useful, it will require adaptation for light vehicles, which is recommended for future research.

This approach and representation helps to make the complexity of collision more explicit than typical narrative investigation reports (Walker et al, 2010). The list of twenty-one potential recommendations addresses the decisions, actions and events at all eight levels of the AcciMap. These show that there is no single, simple, fix for collisions, rather that the whole system needs to be addressed if collisions of this nature are to be reduced. Goode et al. (2018) describe a structured process for developing incident reporting and learning systems based on Rasmussen's framework and AcciMap and ActorMap method (see Figure 5). This process provides a road map for the work that is required to implement Accimap within a national road crash investigation body. The process involves close collaboration with end-users and includes identifying end-user needs (i.e., the analysts that will be using the incident reporting and learning system), developing a suitable classification scheme for Accimap (to ensure reliability and validity), conducting formal end-user evaluations, developing a suitable data collection methodology, developing tools to support learning from incidents, and developing appropriate support tools such as software analysis tools.

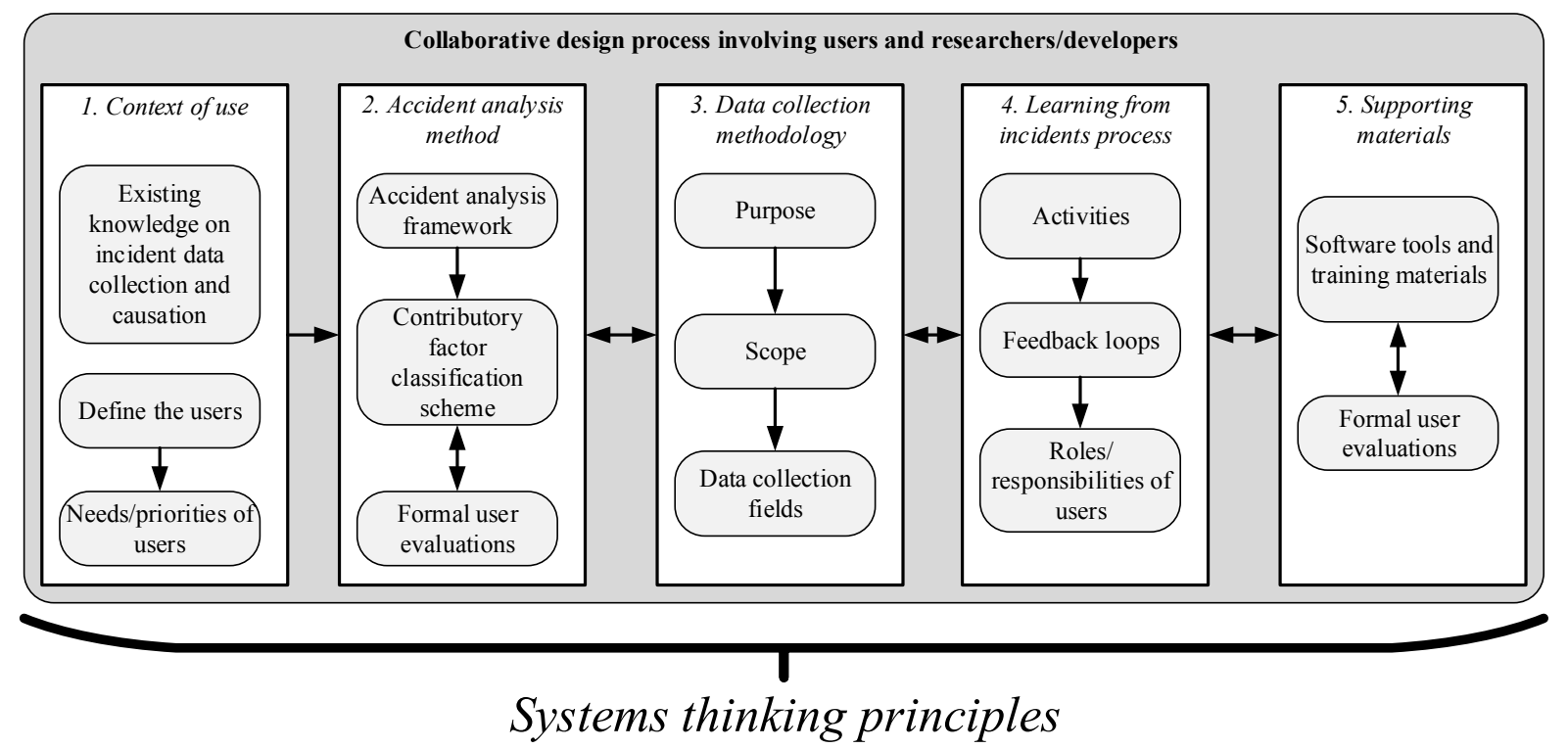

Figure 5: A structured process for developing incident reporting and learning systems

It is accepted that analysing 1,700 annual road deaths in the UK using Actor and AcciMaps will be impossible to resource given current levels of funding. Nevertheless, it is anticipated that major road collisions (involving multiple deaths) and indicative collisions (they type that keep reoccurring) would be prioritised for in-depth investigation by a national body for road safety. Provided that there is a reliable classification scheme for the Actor Maps and AcciMaps 
(discussed previously), one of the major benefits of the approach is the ability to aggregate the data (see Newman et al, 2013). The aggregation of the Actor Maps and AcciMaps with data from multiple collisions should reveal the frequencies that particular actors and events are implicated. This provides the opportunity for strategic interventions to be prioritised, so that maximum benefit for road safety can be realised. Currently the data collected on road collisions tends to focus mainly (although not exclusively) at the bottom two levels of the Actor Map and AcciMap (namely: driving processes, equipment and environment). For the approach to be successful, more effort will need to be put into collecting data at the higher system levels (although these might not change considerably across collisions - but this needs to be formally assessed).

\section{Conclusions}

This paper has sought to present a view on accident analysis methods and their applicability to road collisions. Eight methods were selected for review, and these have been applied to the Uber collision case study, so that their analysis and representation can be better understood. On the basis of the case study, the Actor Map and AcciMap were selected as good candidates for systems analysis of road collisions. To help with the consistency of reporting and aggregation of data, classification schemes need to be developed to support the development of Actor Maps and AcciMaps. A recent study has already presented an Actor Map scheme for the UK (Mcllroy et al., 2018), which would provide a good starting point. In addition, classification schemes have been developed for road freight crashes (Newman and Goode, 2015; Newman et al, 2017). These need to be extended for light vehicles. To improve the usability of the AcciMap method, a matrix for associating events at the same and different levels in the system hierarchy should be developed. This should make it easier for analysts to construct AcciMaps. Studies of the reliability and validity of the Actor Map and AcciMap methods also need to be conducted alongside the training in the approach.

\section{Acknowledgements}

This paper is based on research sponsored by the RAC Foundation for the Road Collision Investigation Project (funded by the Department for Transport in the UK). Prof Stanton contributions to this paper was also supported by the National Institute for Health Research using Official Development Assistance (ODA) funding and the Engineering and Physical Sciences Research Council as part of the TASCC programme: Human Interaction: Designing Autonomy in Vehicles (HI:DAVe), grant reference number EP/N011899/1.

\section{References}

Checkland, P. (1981). Systems Thinking, Systems Practice. Chichester: Wiley. 
DfT (Department for Transport) (2017). Reported road casualties Great Britain 2017. London: Department for Transport. Retrieved from:

https://assets.publishing.service.gov.uk/government/uploads/system/uploads/ attachment_data/file/744078/infographic-2017-annual-report.pdf (12 December 2018).

Eurostat (2017). Air safety statistics in the EU Retrieved from: https://ec.europa.eu/eurostat/statisticsexplained/index.php/Air_safety_statistics_in_the_EU (12 December 2018)

Gooding, S. (2017). Towards an Accident Investigation Branch for Roads? London: RAC Foundation.

Greenwood, M., and Woods, H. M. (1919). The incidence of industrial accidents upon individuals: With special reference to multiple accidents (No. 4). HM Stationery Office [Darling and son, Limited, printers].

Heinrich, H. W. (1931). Industrial Accident Prevention: A scientific approach. New York: McGraw-Hill.

Hendrick, K. and Benner, L. (1987). Investigating Accidents with S-T-E-P. New York: Marcel Dekker.

Hollnagel, E. (2012). FRAM: The Functional Resonance Analysis Method. Farnham: Ashgate.

Hollnagel, E., Woods, D. D., and Leveson, N. (2006). Resilience Engineering: Concepts and Precepts. Farnham: Ashgate.

ICI (1970). Bow-Tie. Retrieved from: https://www.cgerisk.com/knowledgebase/The_history_of_bowtie (12 December 2018).

Kysor, H. D. (1973). Safety management system. Part I: the design of a system. National Safety News, 108, 98-102.

Larsson, P., Dekker, S.W.A. and Tingvall, C. (2010). The need for a systems theory approach to road safety. Safety Science, 48 (9), 1167-1174.

Leveson, N.G. (2004). A new accident model for engineering safer systems. Safety Science, 42 (4), 237-270.

Leveson, N.G. (2012). Engineering a Safer World: Applying Systems Thinking to Safety. Boston: MIT Press

McIlroy, R. C., Plant, K. L., Hoque, M. S., Wu, J., Kokwaro, G. O., Vũ, N. H. and Stanton, N. A. (2018). Who is responsible for global road safety? A cross-cultural comparison of Actor Maps. Accident Analysis and Prevention, 122, 8-18. 
MAIB (2018). Annual Report 2017 Publication date: June2018. Southampton: Marine Accident Investigation Branch. Retrieved from:

https://assets.publishing.service.gov.uk/government/uploads/system/uploads/ attachment_data/file/720443/MAIB_Annual_Report_2017.pdf (12 December 2018).

Newnam, S. and Goode, N. (2015). Don't blame the driver: A systems analysis of the causes of road freight crashes, Accident Analysis and Prevention, 76, 141151.

Newnam, S., Goode, N., Salmon, P. M. and Stevenson, M. (2017). Reforming the road freight transportation system using systems thinking: An investigation of Coronial inquests in Australia, Accident Analysis and Prevention, 101, 28-36.

NTSB (2018) National Transportation Safety Board Preliminary Report Highway: HWY18MH010. Retrieved from:

https://www.ntsb.gov/investigations/AccidentReports/Pages/HWY18MH010prelim.aspx (12 December 2018).

ORR (2017) Office of Rail and Road - Rail Safety Statistics 2016-17 Annual Statistical Release. Publication Date: 26 September 2017. London: Office of Rail and Road.

Parnell, K., Stanton, N. A. and Plant, K. L. (2017) What's the law got to do with it? Legislation regarding in-vehicle technology use and its impact on driver distraction. Accident Analysis and Prevention, 100, 1-14.

Perrow, C. (1984). Normal accidents: Living with high risk systems. New York: Basic Books.

Rasmussen, J. (1997). Risk management in a dynamic society: A modelling problem. Safety Science, 27, 183-213.

Reason, J. (1990). Human Error. Cambridge: Cambridge University Press.

Robinson, G.H. (1982). Accidents and sociotechnical systems: principles for design. Accident Analysis and Prevention, 14 (2), 121-130.

Salmon, P. M., Cornelissen, M. and Trotter, M. J. (2012) Systems-based accident analysis methods: A comparison of Accimap, HFACS, and STAMP. Safety Science, $50,1158-1170$

Salmon, P. M., Goode, N., Taylor, N., Dallat, C., Finch, C. and Lenne, M. G. (2017). Rasmussen's legacy in the great outdoors: a new incident reporting and learning system for led outdoor activities. Applied Ergonomics, 59, 637-648.

Salmon, P. M and Lenné, M. G. (2015). Miles away or just around the corner? Systems thinking in road safety research and practice. Accident Analysis and Prevention, 74, 243-249. 
Shappell, S. A. and Wiegmann, D. A. (2001). Applying reason: The human factors analysis and classification system (HFACS). Human Factors and Aerospace Safety, 1(1), 59-86.

Stanton, N. A. (2016). On the Reliability and Validity of, and Training in, Ergonomics Methods: A Challenge Revisited. Theoretical Issues in Ergonomics Science, 17 (4), 345-353.

Stanton, N. A. and Harvey, C. (2017). Beyond human error taxonomies in assessment of risk in sociotechnical systems: a new paradigm with the EAST 'broken-links' approach. Ergonomics, 60 (2) 221-233.

Stanton, N. A., Rafferty, L. A. and Blane, A. (2012). Human Factors analysis of accidents in System of Systems, Journal of Battlefield Technology, 15 (2), 23-30.

Stanton, N. A., Salmon, P. M., Rafferty, L. A., Walker, G. H., Baber, C. and Jenkins, D. (2013) Human Factors Methods: A Practical Guide for Engineering and Design (second edition). Ashgate: Aldershot.

Stanton N. A., Salmon P. M. and Walker G. H. (2019). Systems Thinking in Practice: Applications of the Event Analysis of Systemic Teamwork Method. CRC Press: Boca Raton, USA.

Stanton, N.A. and Young, M.S. (1998). Is utility in the mind of the beholder? A study of ergonomics methods. Applied Ergonomics, 29, 1, 41-54.

Stanton, N.A. and Young, M.S. (1999a). What price ergonomics? Nature, 399, 197-8.

Stanton, N. A., and Young, M. S. (1999b). A Guide to Methodology in Ergonomics: Designing for Human Use. London: Taylor \& Francis.

Stanton, N. A. and Young, M. S. (2003). Giving ergonomics away? The application of ergonomics methods by novices. Applied Ergonomics, 34, 479490.

Stanton, N. A., Young, M. S. and Harvey, C. (2014). A Guide to Methodology in Ergonomics: Designing for Human Use (second edition). Taylor \& Francis: London.

Sterman, J.D. (2000). Business Dynamics: Systems Thinking and Modeling for a Complex World. Irwin McGraw-Hill, Boston.

Svedung, I. and Rasmussen, J. (2002). Graphic representation of accident scenarios: Mapping system structure and the causation of accidents. Safety Science, 40, 397-417. 
Talbot, R., Fagerlind, H., \& Morris, A. (2013). Exploring inattention and distraction in the SafetyNet Accident Causation Database. Accident Analysis \& Prevention, 60, 445-455

Taylor, F. W. (1911). The Principles of Scientific Management. New York: Harper.

Trist, E. L. and Bamforth, K. W. (1951). Some social and psychological consequences of the longwall method of coal-getting: An examination of the psychological situation and defences of a work group in relation to the social structure and technological content of the work system. Human Relations, 4(1), 3-38.

Underwood, P. and Waterson, P. (2014). Systems thinking, the Swiss Cheese Model and accident analysis: a comparative systemic analysis of the Grayrigg train derailment using the ATSB, AcciMap and STAMP models. Accident Analysis and Prevention, 68, 75-94

Von Bertalanffy, L. (1950). An outline of general system theory. British Journal for the Philosophy of Science, 1, 134-165.

Walker, G. H., Stanton, N. A., Baber, C., Wells, L., Gibson, H., Salmon, P. M. and Jenkins, D. P. (2010). From ethnography to the EAST method: A tractable approach for representing distributed cognition in air traffic control. Ergonomics, 53 (2), 184-197.

Warmerdam, A., Newnam, S., Sheppard, D., Griffin, M. and Stevenson, M. (2017). Workplace road safety risk management: An investigation into Australian practices. Accident Analysis \& Prevention, 98, 64-73.

Watson, H. A. (1962). Fault Tree Analysis. Retrieved from: https://en.wikipedia.org/wiki/Fault_tree_analysis\#cite_note-8

(12 December 2018) 


\section{Appendix A: Description of the Methods (supplementary materials)}

A description of each of the eight methods being considered in the evaluation is presented in alphabetical order with a brief description and the accompanying model.

ACCIMAP is based on Rasmussen's (1997) Risk Management Framework. He used a road traffic accident to demonstrate the AcciMap analysis, giving the example of an oil truck, which crashed, disgorging oil into a reservoir. Accimap is a generic approach, which has been widely used in many different domains. Normal variations in behaviour (rather than exceptions) are seen to result in accidents within the system. The method identifies and links contributory failures (both top-down and bottom-up) across six levels of the sociotechnical system (government, regulators/associations, company, management, staff, work) so that countermeasures can be put in place (see Figure A.1).

The strengths of this approach is that it is holistic, describing failures across the system and allowing measures to be identified to ameliorate for this. The lack of taxonomies makes it flexible to fit different domains.

There are weaknesses, however. It does not specifically identify cognitive factors, and it highlights decisions rather than the factors influencing those decisions. The lack of taxonomies means that it is dependent on the subjective judgment of the analyst, which may impact on its reliability. Moreover, without taxonomies, aggregate analysis of multiple accident cases becomes more difficult. The output in diagrammatic form is quite complex. 
Figure A.1: The AcciMap model
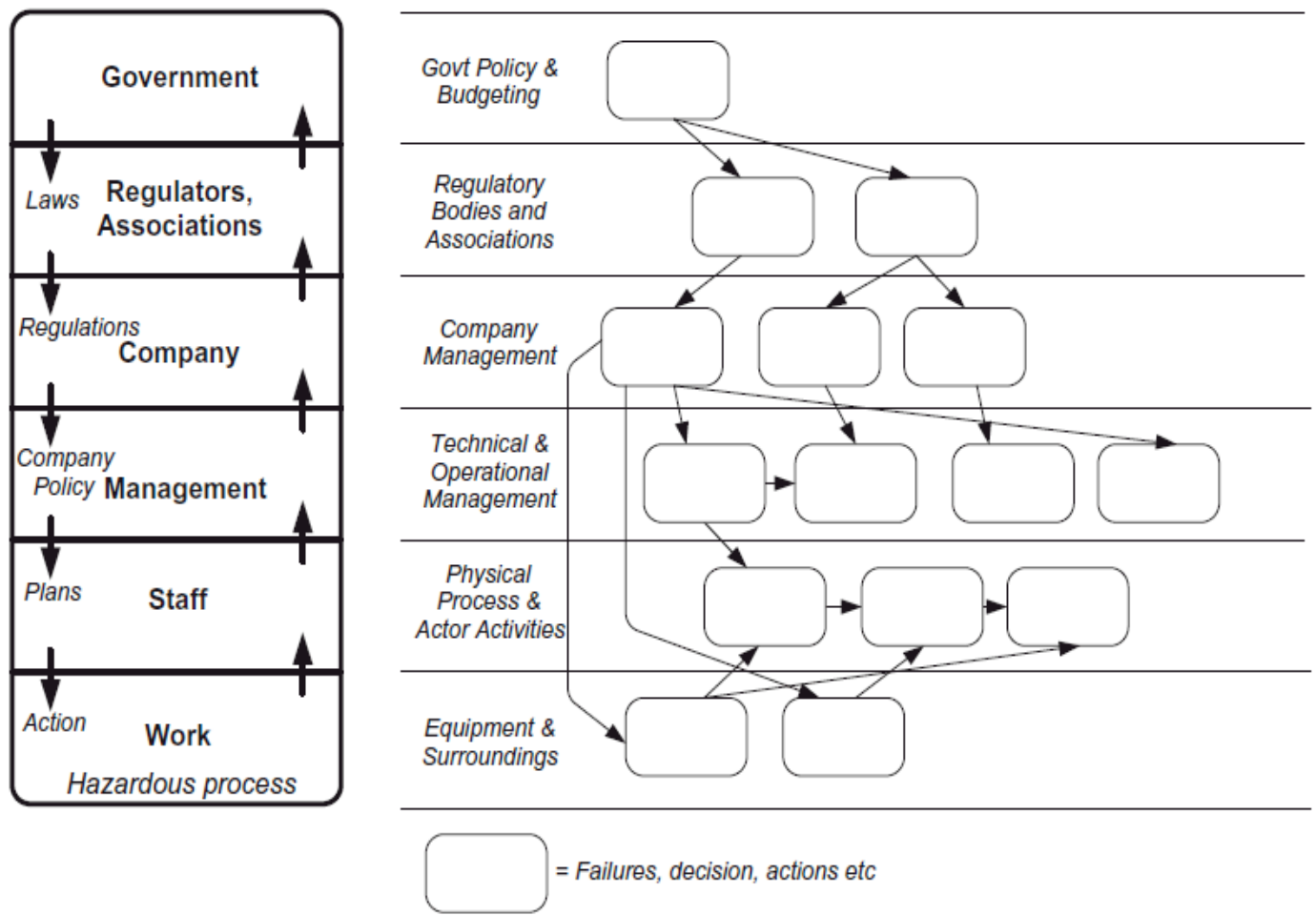

Source: Svedung \& Rasmussen (2002)

BOW-TIE has its origins in process engineering. It shows the relationship between the causes and consequences of an event in a Bow-Tie diagram (see Figure A.2) - so called owing to its shape, which obviously resembles a bow tie. In the centre of the Bow-Tie diagram is the hazardous event (HE). The left wing of the bow uses Fault Tree Analysis to show the relationship between the possible causes (and possible mitigating control and recovery safety measures, current or planned) of an event. The right wing of the bow uses event tree analysis to show the relationship to the consequences (and possible recovery measures, current or planned) that may follow the event. Thus the Bow-Tie method generates a diagram that identifies: the HE, the causes that may lead to the $\mathrm{HE}$, the consequences that may result from the HE, and the safety measures that may change the likelihood of the causes (proactive safety measures) or the consequences (reactive safety measure) of the HE.

The strength of the model is that it gives a clear causes/consequences diagram of the incident and it can be used to model likely scenarios.

The weaknesses are that it is a sequential model, and as such does not show interactions between the risk factors; moreover, it does not take into account the higher levels, such as policy and regulation. 
Figure A.2: The Bow-Tie model

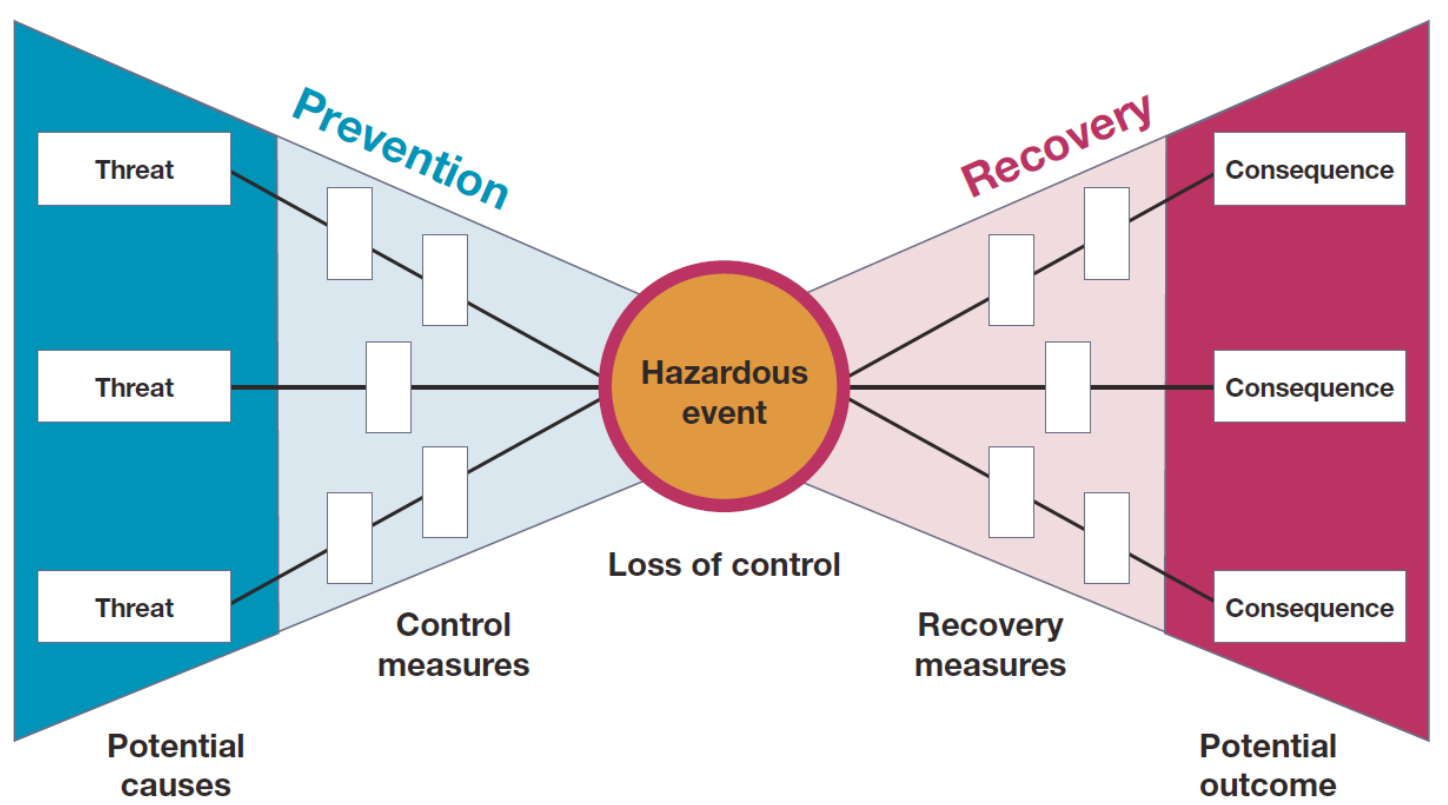

Source: https://slideplayer.com/slide/12655534 (slide 8)

EAST-BL (Event Analysis of Systemic Teamwork - Broken Links) views accidents as a consequence of variability in human performance, leading to a failure to communicate information via social and task networks (see Figure A.3). EAST analyses the communication of information in the system, providing a clear visual representation of the system, the key agents in decisionmaking, and the relationship between individual components. When applying EAST analysis, the social, task and information systems are developed separately and then combined into a network diagram. The first step is to conduct an AcciMap analysis, placing subsystems within the sociotechnical systems (STS) at different levels, showing the links for decision-making and communications between related nodes. In this way, network diagrams are constructed showing the main agents and their relationships. These allow quantitative SNA (social network analysis) metrics to be calculated. For each EAST network, key nodes (i.e. those with the largest number of connections) and key agents are identified. EAST analysis can be used to analyse events prospectively (Broken Links) or retrospectively (Broken Nodes). The analysis in this document is undertaken using broken-node analysis (as it is an event that has already taken place). Broken nodes are failures of the nodes in the social, task and information networks. Broken nodes are identified and the consequences are analysed.

The strengths of EAST-BN (Event Analysis of Systemic Teamwork - Broken Nodes) is that it provides an integrated and holistic approach to interrogating the social, task and information aspects of the STS. It provides a comprehensive model of nodes and links with information flow that can be applied in any domain.

The weaknesses is that diagrams can be complex. 


\section{Figure A.3: The EAST model}

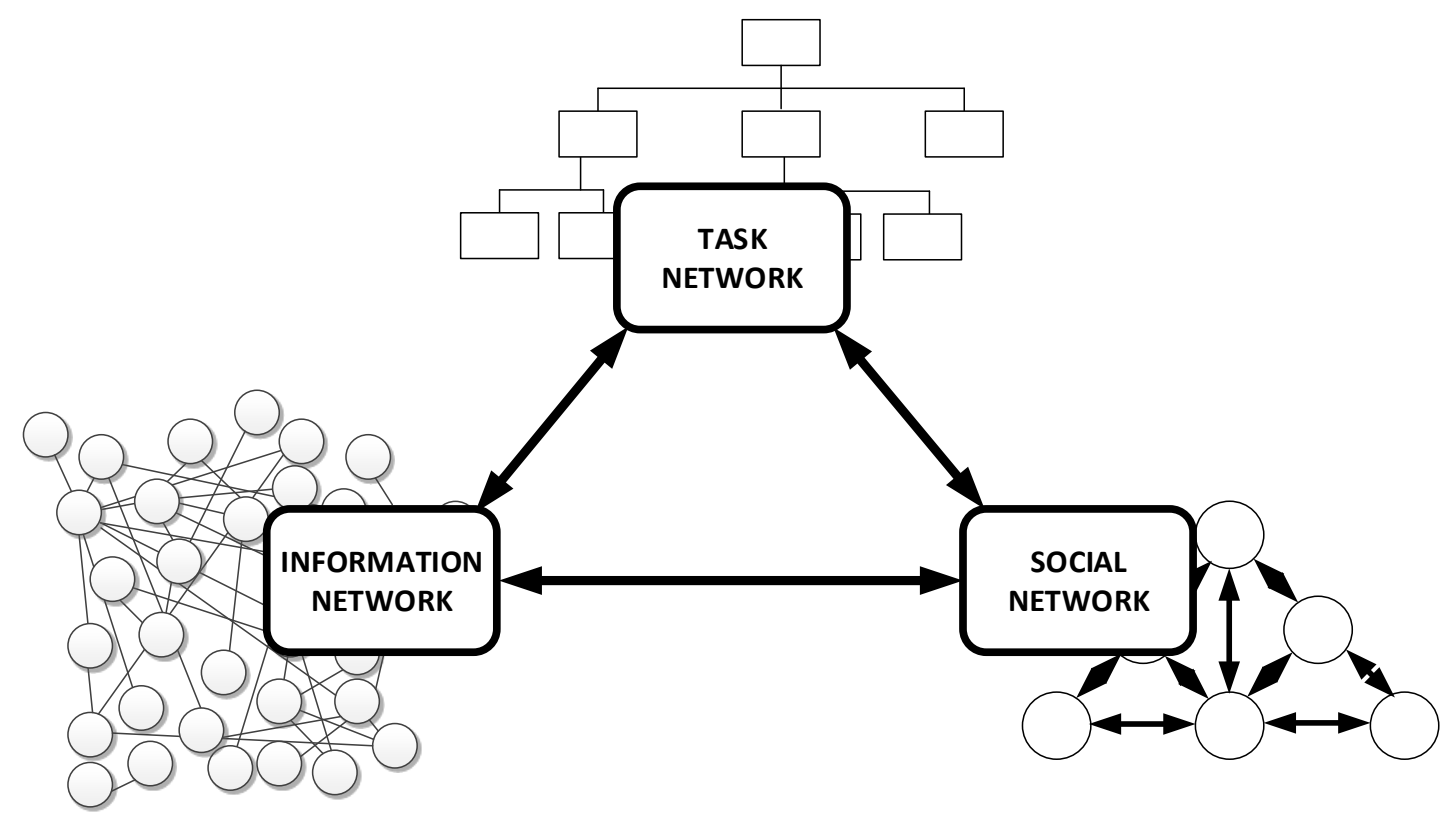

FTA (Fault Tree Analysis) provides a visual representation that identifies and analyses the risk factors involved in a particular incident or event. FTA can be used pre-event to look at possible causes and mitigating measures, and postevent to analyse accidents that have happened. The first step in FTA is to establish a top event (see Figure A.4). All the possible ways that this event could have occurred are then listed. Each option is investigated, using 'AND'/'OR' gates to link the events into a tree. The gates represent ways in which human/machine interactions can produce events. 'AND' gates mean that both events need to occur for the output event to occur, while 'OR' gates mean that only one is necessary. Then a Boolean expression is used to determine 'cut sets'. These are the components in a system which, when they fail, result in system failure.

The strength of an FTA is that it is a sequential model describing and analysing the events leading up to an incident and highlighting countermeasures that could be put in place to ameliorate this.

The weaknesses are that continuous and concurrent events are hard to represent in the model, and the interactions between components are not adequately shown. Further, FTA does not take a systems approach and could be said to be reductionist in understanding risk and safety management, if one accepts the premise that a complex system cannot be understood simply by looking at the system's component parts. 


\section{Figure A.4: The fault tree model}

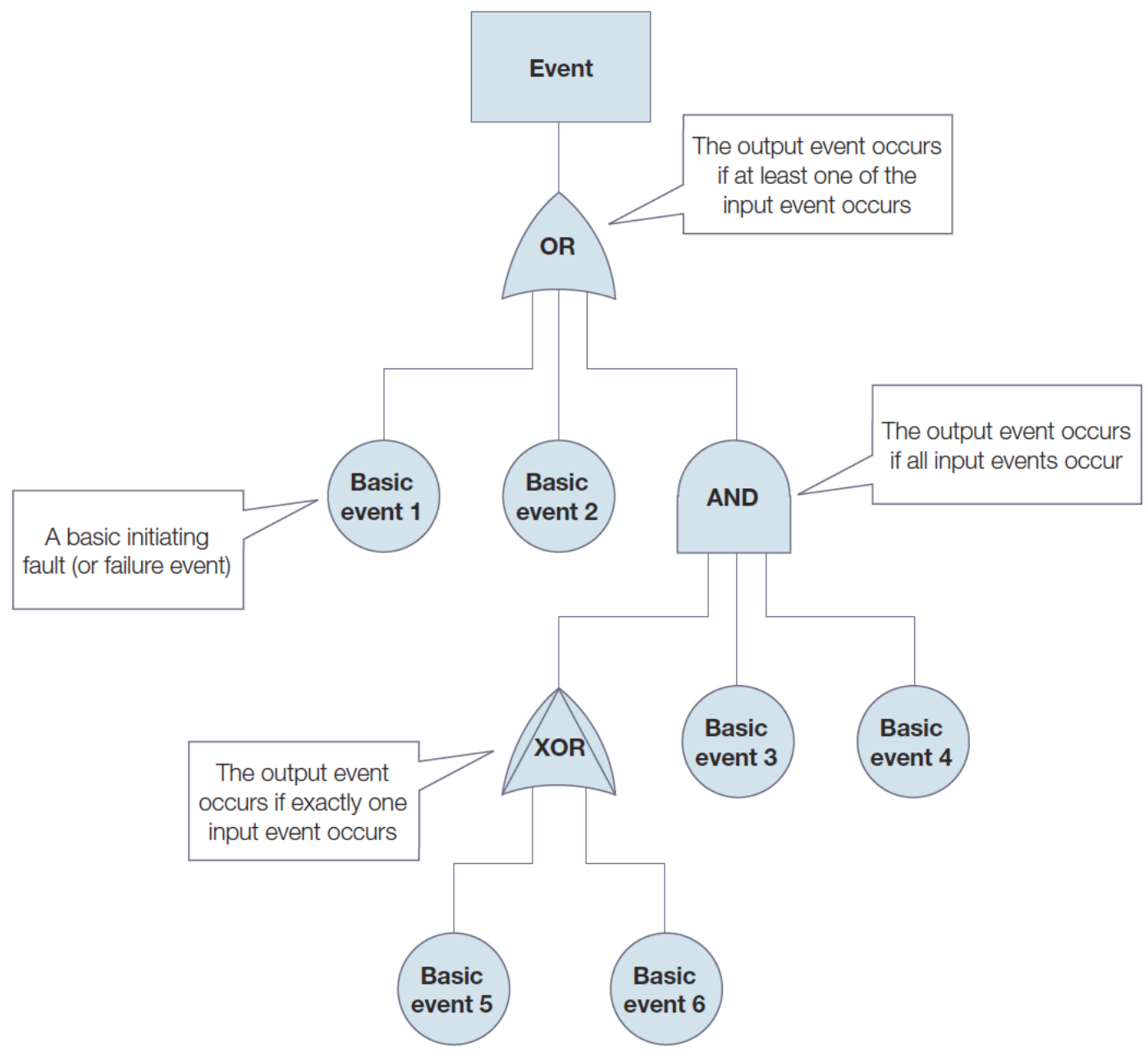

Source: https://conceptdraw.com/a362c3/p1/preview/256

FRAM (Functional Resonance Analysis Method) is based on the principle that variability in performance is both normal and necessary, also that the combination of normal variability can lead to unexpected consequences that can exceed normal limits and result in a critical incident. The FRAM method develops a diagrammatic model of the system and system behaviour illustrating the dynamic nature of interactions. Where functions interact they are described as 'couplings' (see Figure A.5). Couplings may occur only under certain circumstances. It could be that normal variability on its own may not create a problem, but when it occurs alongside other variability it can lead to excessive variability and an incident occurs. The FRAM method entails first identifying and describing the system functions, then specifying the variability and links between functions. Finally measurers/barriers that monitor or reduce unwanted variability are highlighted.

The strengths of FRAM are that it looks at normal performance and its variability. It also considers interactions between functions and their cumulative affect. In this way it takes a systems approach to provide the what, when, how and why of the incident. It can be used to identify potential risks and countermeasures to put in place, as well as after an event to identify causes. 
One weakness of FRAM is that it does not consider the higher levels of functioning such as government and regulatory bodies.

\section{Figure A.5: The couplings model}

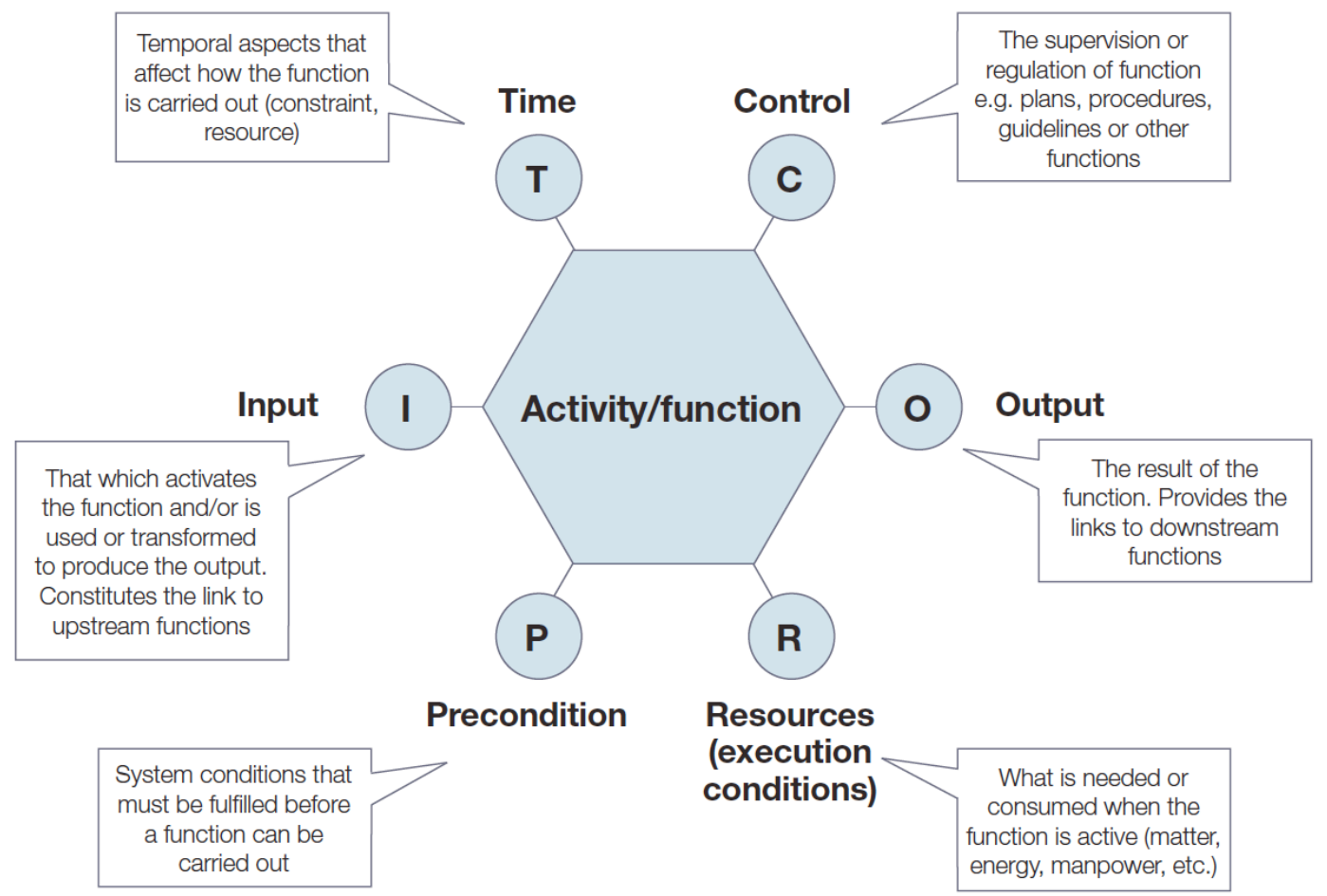

Source: http://functionalresonance.com/onewebmedia/Manual\%20ds\%201.docx.pdf

HFACS (Human Factors Analysis and Classification Scheme) is a taxonomy-based accident analysis method, initially developed for the aviation industry. It is based on Reason's Swiss Cheese Model. It classifies and links failures across four levels (see Figure A.6). It identifies human and system contributions so that countermeasures can be deployed to prevent or reduce further accidents. HFACS protocol begins with the analysis of primary reports. Data is categorised according to the taxonomy.

The strengths of taxonomy-based methods is that they make themes in causal factors easy to identify, and also enhance reliability. They lend themselves to multiple-case analyses. A visual representation is produced which is clear and easy to read.

The weaknesses is that taxonomies can constrain the classification to certain types of failures, and they need to be developed to be domain-specific. Having only four levels means that higher levels, such as government and regulators, are not considered; however, more recent analyses have extended HFACS to include 'government' and 'other' categories. 
Figure A.6: The HFACS model

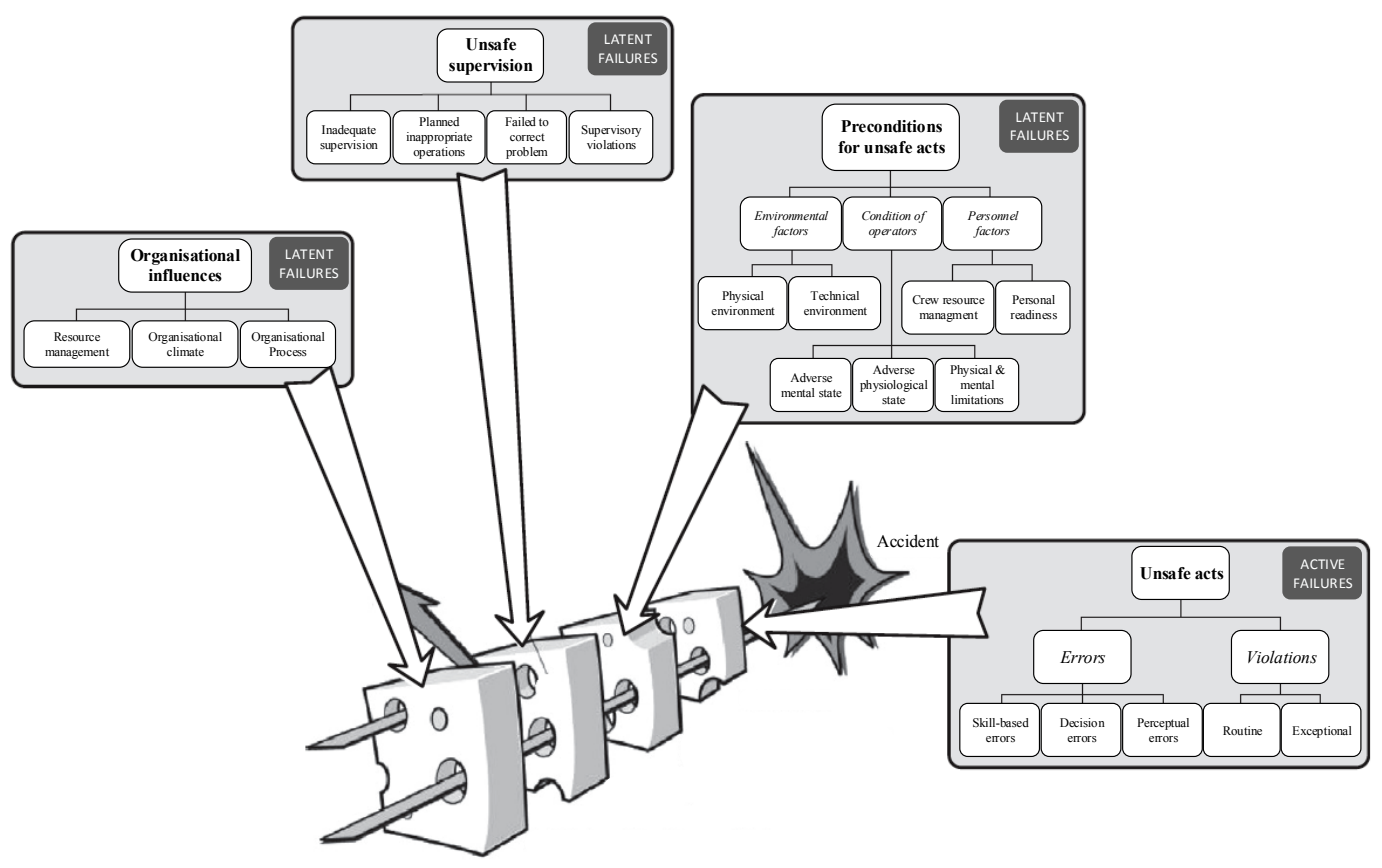

STAMP (Systems-Theoretic Accident Model and Processes) is based on Sociotechnical Systems (STS) Theory, which is generic rather than domainspecific. It can be used for accident analysis post-event and/or risk analysis preevent, and provides an overview whereby systems are seen as having hierarchical levels, each with a control structure. Controls enforce constraints, resulting in safe behaviour. Controls and constraints operate bottom-up and topdown between levels. Rather than accidents being viewed as the consequence of events, they are seen as resulting from control failures (i.e. inadequate enforcement of constraints or lacking/inaccurate constraints). The system must have an adaptive feedback mechanism of control and information. SystemsTheoretic Accident Model and Processes- Causal Analysis using Systems Theory (STAMP-CAST) proposes a taxonomy of control failures using observations and scenarios as data collection methods. It accounts for cognitive factors by considering the context for decisions and including a 'mental model flaws' category. The first step in carrying out a STAMP-CAST analysis is to model levels of the STS (see Figure A.7). Next, the potential or actual accidents to be considered, and the control flaws and hazards are identified (see Table A.1). Then a model of the functional control structure is created, specifying potential unsafe control actions and how they could occur. Lastly, remedies are suggested. The output consists of two stages: the control structure and then a more detailed analysis of key personnel selected from the control structure.

The strengths of constructing a control structure diagram is that a deeper representation of the system is developed, which provides a more comprehensive understanding than linear models and allows modelling of future scenarios to aid accident prevention. The whole system, including social and organisational factors, is considered, with the interactions that lead to accidents 
being identified. Thus appropriate countermeasures can be specified and put in place.

The weaknesses of this approach are the time taken and knowledge required to construct the control structure diagram. Moreover, it can be more suited to technical and control failures, with environmental factors being more challenging to fully consider, and complex human and organisational factors being harder to place. It also assumes a hierarchical model but relationships between the agencies and agents may not necessarily be hierarchical.

Figure A.7: The STAMP model
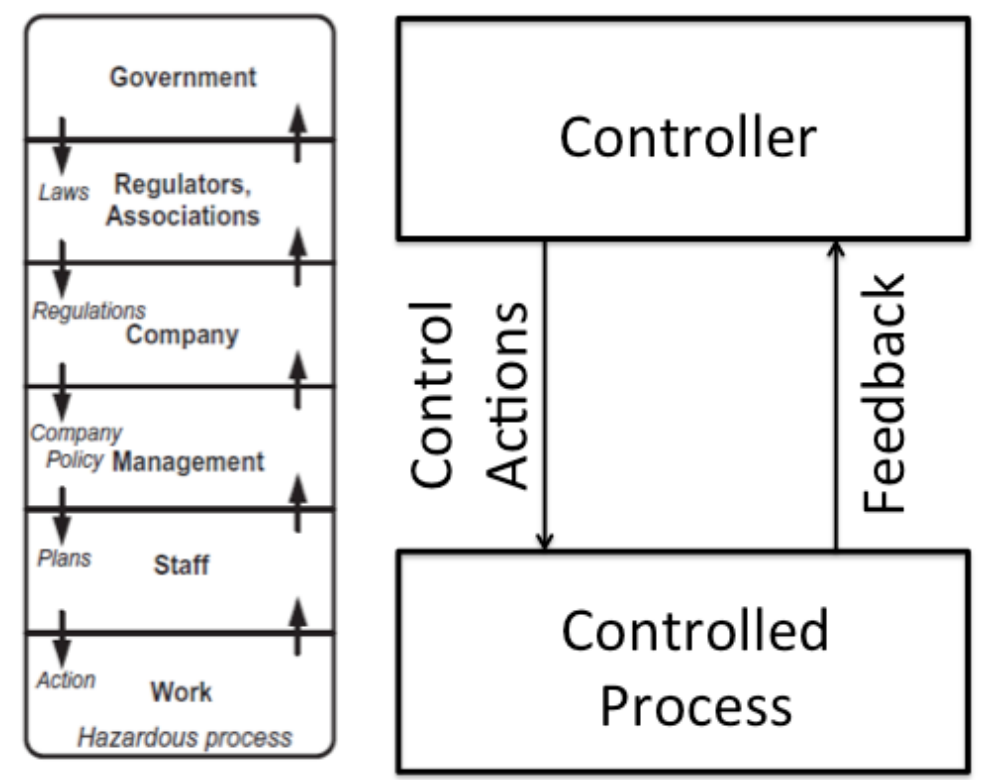

Table A.1: Classification of key hazards in STAMP-CAST

\begin{tabular}{|c|c|}
\hline Control flaw & Hazard \\
\hline $\begin{array}{l}\text { Inadequate } \\
\text { enforcement of } \\
\text { constraints (control } \\
\text { actions) }\end{array}$ & $\begin{array}{l}\text { - } \text { Unidentified hazards } \\
\text { - Design of control algorithm (process) does not } \\
\text { enforce constraints } \\
\text { - Inadequate co-ordination among controllers and } \\
\text { decision-makers }\end{array}$ \\
\hline $\begin{array}{l}\text { Inadequate } \\
\text { execution of control } \\
\text { action }\end{array}$ & $\begin{array}{l}\text { - } \text { Communication flaw } \\
\text { - Inadequate actuator operation } \\
\text { - Time lag }\end{array}$ \\
\hline $\begin{array}{l}\text { Inadequate or } \\
\text { missing feedback }\end{array}$ & 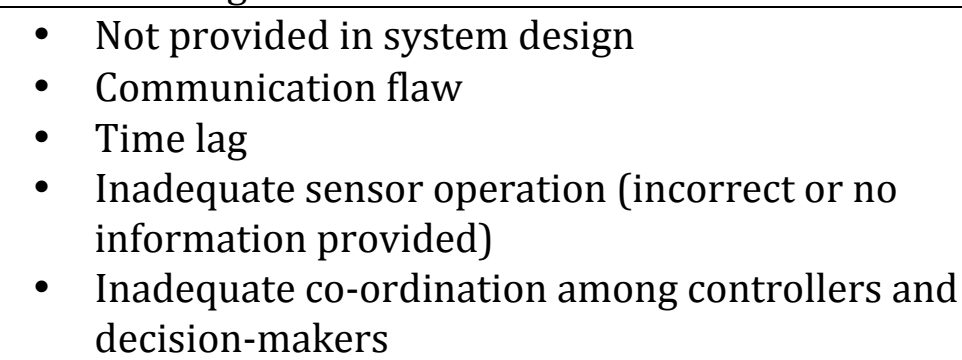 \\
\hline
\end{tabular}


STEP (Sequential Timed Event Plotting) is a sequence model that plots a timeline of events from the start to finish of a critical incident. Each actor is specified on the left-hand axis of a STEP worksheet with time running along the y-axis (see Figure A.8). Each event/action performed by an actor is represented by a 'building block', which has information on the time, duration, agent, event/action and source. Directional arrows show the relationships between events. In this way, STEP provides a visual representation of the events in a logical order, showing events happening in sequence or in parallel and the nature of any interaction between them. Once the worksheet has been completed, the backSTEP technique is used to work from end to start of the incident and ensure that all events are listed and the building blocks are placed accurately. The worksheet is then analysed to identify safety problems (represented by a triangle) and specify countermeasures.

The strengths of STEP are that it is relatively simple to use and offers a clear description and analysis of the 'what happened when' so that countermeasures can be identified and put in place. By highlighting the interactions in the incident it demonstrates the impact of one agent/event on another.

Weakness, are that it is limited in its scope. It does not represent workplace, management, regulatory or government factors, and it does not offer a systems analysis of the critical incident.

\section{Figure A.8: The STEP model}

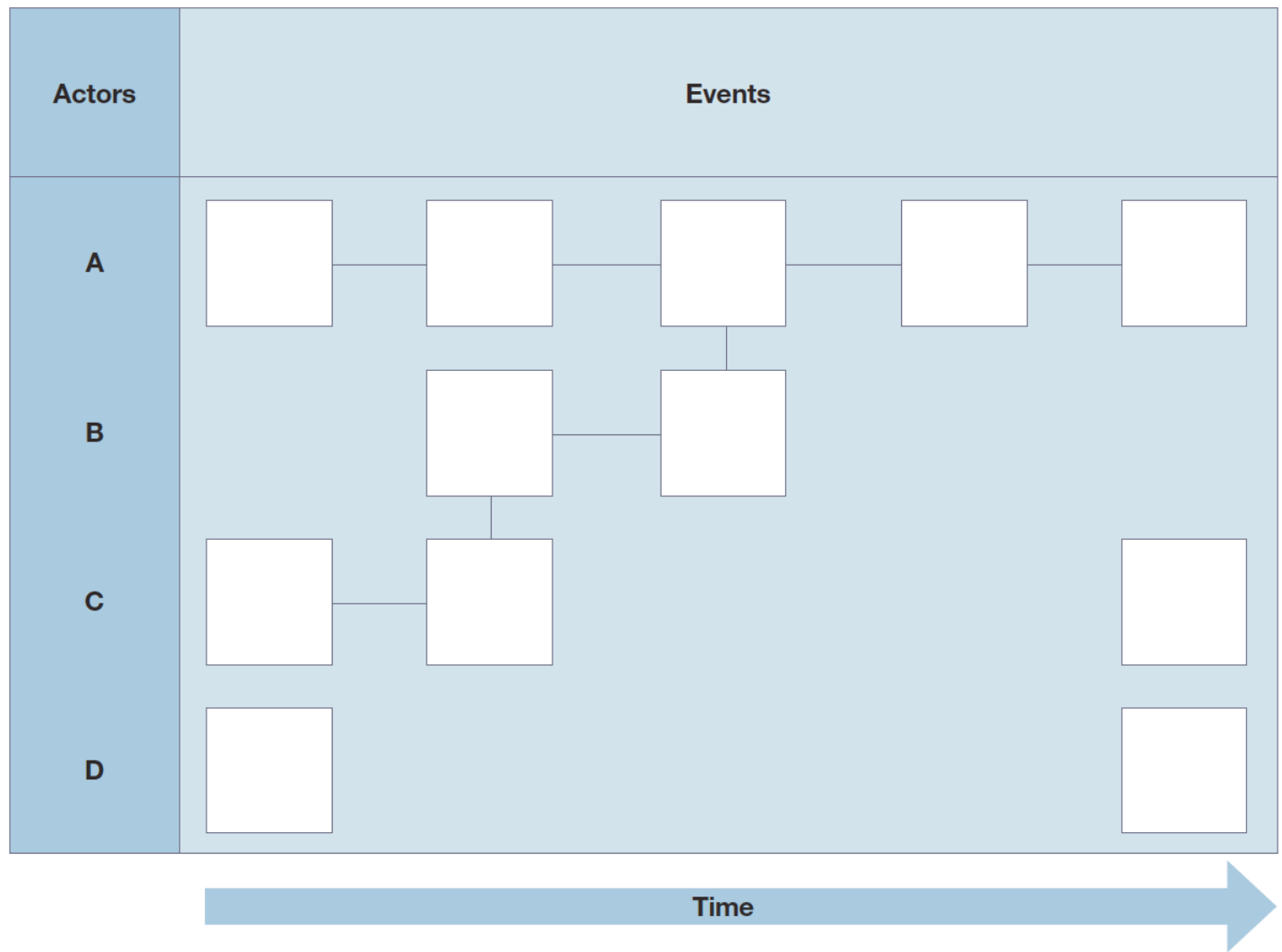




\section{Appendix B: Case Studies Applying the Methods to the Uber Collision (supplementary materials)}

The case studies are presented in alphabetical order of the method name. Each is accompanied by a brief description of the way in which the method represents the Uber collision with the pedestrian.

ACCIMAP represents the events, failures, decisions and actions that interacted to create the Uber collision are presented in the boxes in Figure B.1, with the interactions between them indicated by the arrows. At the top of the AcciMap, the lack of international and national standards for automation design and testing meant that Uber had no technical guidance for appropriate interfaces, safety standards, or testing regimes. The task of the Uber test driver required them to look at the road scene, evaluate the performance of the vehicle, and make notes as required on the tablet. The driver looked up approximately half a second before the collision and, on spotting the pedestrian wheeling a bicycle across the road (taking a direct route to a homeless shelter), she grabbed the steering to attempt a swerve. Although the vehicle automation had identified the pedestrian (on its third attempt) and activated the AEB, it did not respond because the Uber engineers had disabled it. The pedestrian was struck at a speed of $39 \mathrm{mph}$ and died in a local hospital. It was also noted that the pedestrian was not crossing the road at the pedestrian crossing. Although the crossing had the appearance of a pedestrian crossing, there were small, unlit, signs stating that the real crossing was further up the road. It is possible that the pedestrian may not have seen the signs (as there was no roadway lighting). It is equally possible that a strong 'desire line' existed in this location, which would render the appeals from signs ineffective in any case. The autopsy revealed that the pedestrian was intoxicated with methamphetamine and marijuana.

Figure B.1: AcciMap of the Uber collision with a pedestrian 


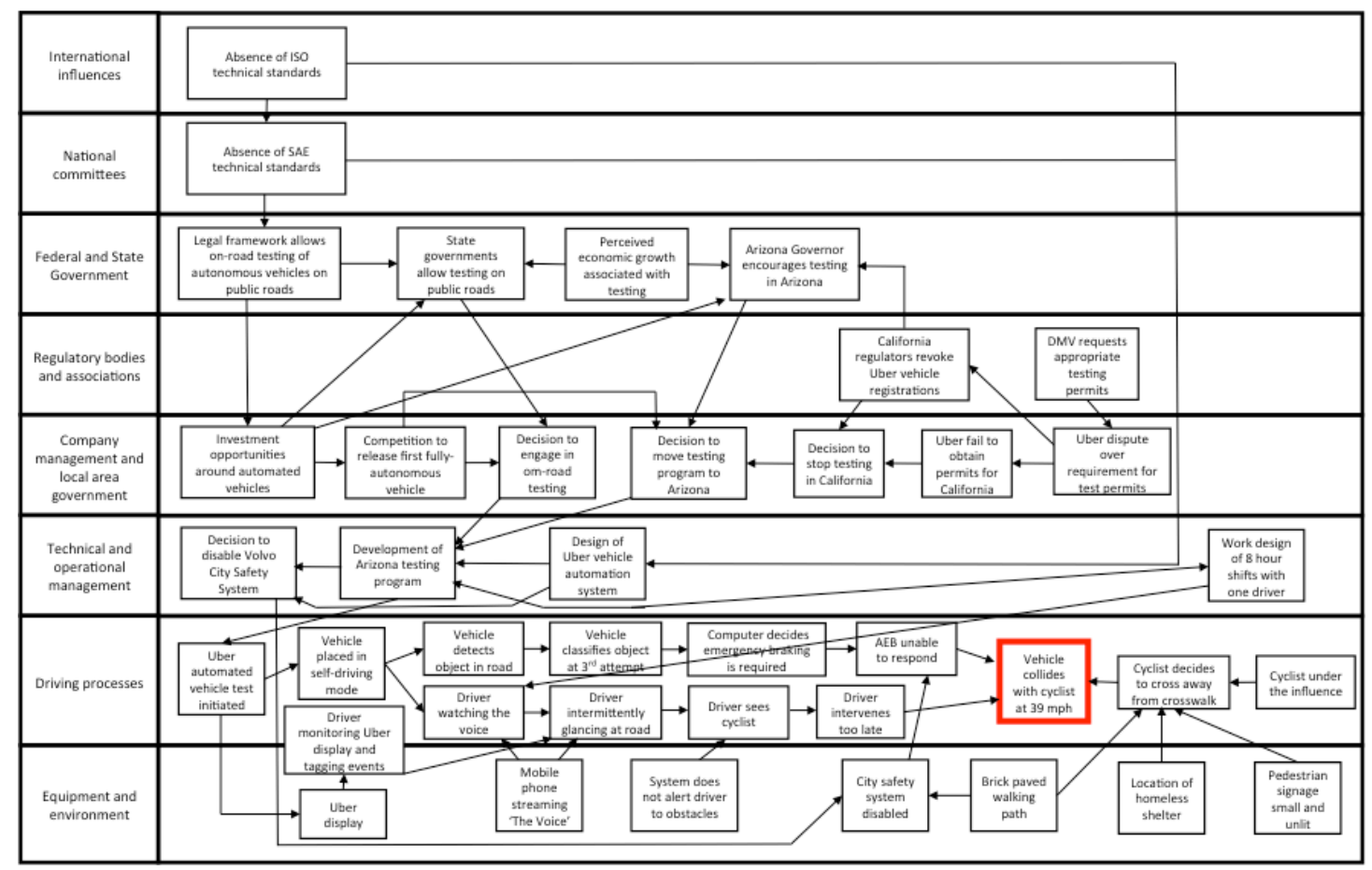

Sources: As table 7 and https://www.theguardian.com/technology/2018/mar/28/uber-arizonasecret-self-driving-program-governor-doug-ducey and https://www.bbc.com/news/technology-44243118 and https://www.citylab.com/transportation/2018/03/former-uber-backup-driver-we-saw-thiscoming/556427/

BOW-TIE represents the threats on the left-hand side of Figure B.1 (i.e. the intoxicated pedestrian, the pedestrian crossing the road at an inappropriate point, the disablement of the Volvo City Safety system by the Uber engineers, the unlit street, the driver scanning the road ahead only intermittently, and the driver allegedly watching The Voice on a smartphone). The corresponding system defences are highlighted in red after each of the threats. These defences, if activated, are supposed to prevent each of the threats from leading to a hazard (which is represented in the circle in the centre of the figure, i.e.: failure to detect pedestrian).

None of the defences worked in this case. Potential recovery mechanisms are presented before the consequences as they could, if activated, mitigate the outcome. In this case, although the Uber system did eventually detect and classify the pedestrian, it was unable to activate the AEB system because it had already been disabled by the Uber engineers. This led to the consequences: the collision between the Uber vehicle and the pedestrian, the suspension of the Uber testing programme, and the police considering whether the driver should be prosecuted. 
Figure B.1: Bow-Tie analysis of the Uber collision with a pedestrian

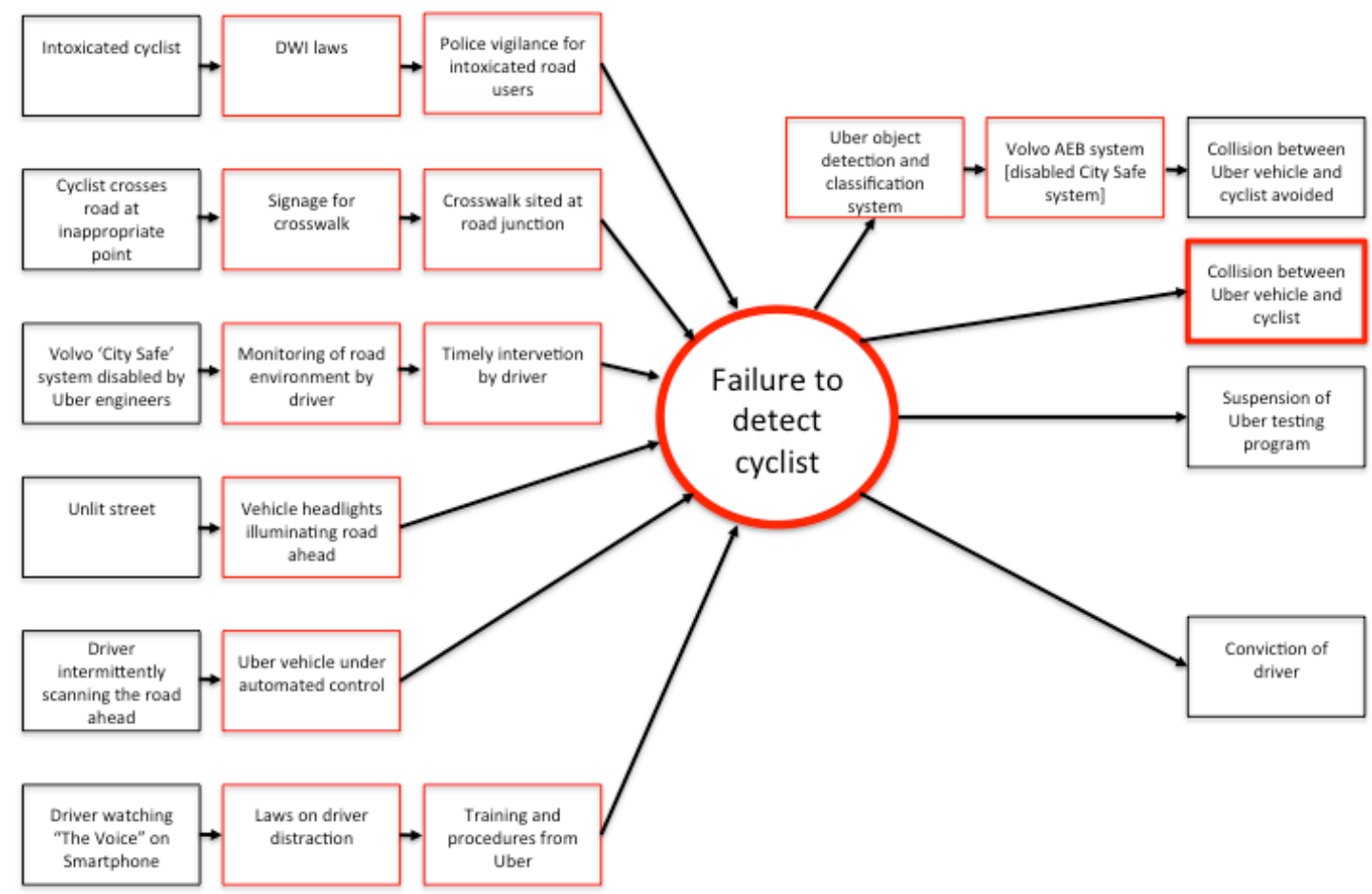

EAST-BN (Event Analysis of Systemic Teamwork - Broken Nodes) represents the task (bottom left), information (top right) and social (bottom right) networks for the Uber collision analysis in Figure B.2. (Recall that the BN - 'Broken Nodes' - version of this method is used to analyse retrospectively.) The broken nodes are represented by the red dashes in the three networks. A broken node is one that is performing suboptimally. There were $9 / 16$ broken nodes in the task network, 8/26 broken nodes in the information network and 5/19 broken nodes in the social network.

For example, in the social network (bottom right of Figure B.2), the pedestrian did not obey the no-crossing sign, but the no-crossing sign was small and unlit. Similarly, the vehicle did not brake for the pedestrian, but the AEB system had been disabled.

In the task network (bottom left of Figure B.2), the pedestrian did not read the sign and find a safe place to cross, nor check the road for traffic. The vehicle did not monitor the driver's alertness, nor provide them with warnings when the need to take manual control arose. Similarly, the driver did not monitor the driving environment or behaviour of the vehicle adequately, nor did they take over manual control before the collision was unavoidable.

In the information network (top right of Figure B.2), the pedestrian did not use the information from the signage to cross further up the road. The Uber vehicle automation system had problems in classifying the pedestrian, first classifying it as unknown, then as a car and finally as a bicycle. With the obstacle detected, it could not evoke the AEB as it had been disabled. Finally, it has been alleged that 
the driver was attending to The Voice rather than the road environment, which led to a very late detection of the pedestrian.

Figure B.2: EAST-BN analysis of the Uber collision with a pedestrian

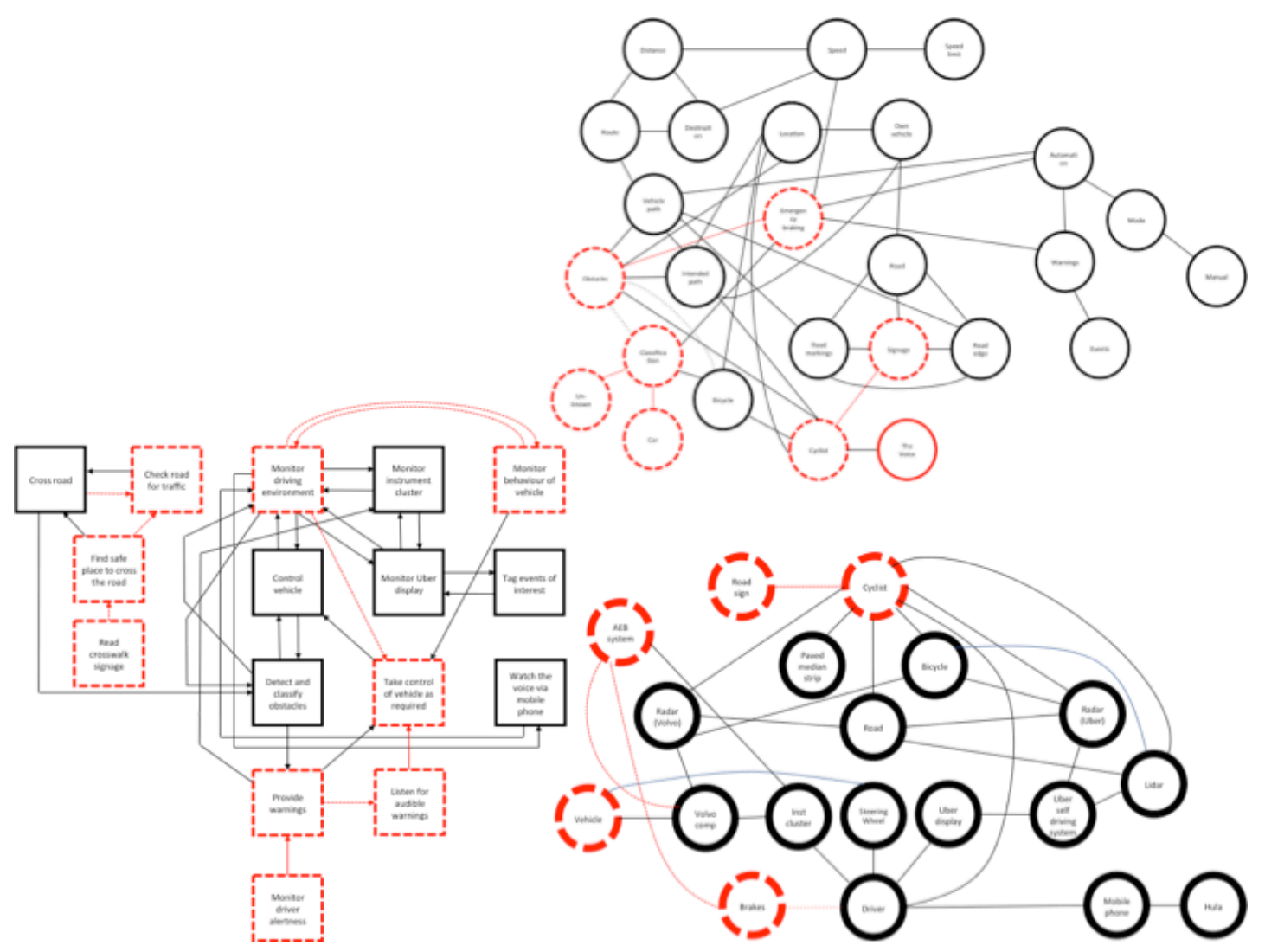

FTA (Fault Tree Analysis) all of the immediate factors present that led to the collision between the Uber vehicle and the pedestrian (which is the top event in Figure B.3). As this analysis is representing a real event, there are no 'OR' gates, just 'AND' gates.

In this case, the pedestrian chose to cross the road at an inappropriate point, which was probably due to a combination of being under the influence of drugs, the paved central reservation having the appearance of a pedestrian crossing, the pedestrian crossing signage being small and unlit, and the paved area being in the desire line to the homeless shelter (the path the pedestrian was taking). The AEB failed to activate because it had been disabled by the Uber engineers (because they thought the Volvo City Safety system would trigger at false obstacles and cause erratic driving of the autonomous vehicle). Finally, the driver intervened too late to prevent the collision, which was probably due to a combination of allegedly watching The Voice on a smartphone, being required to monitor the Uber display on the centre console, and the absence of a warning to alert the driver to the presence of an obstacle in the path of the vehicle. The reasons why the driver was allegedly watching The Voice on a smartphone are probably related to the design of eight-hour shifts in an automated vehicle with repetitive work, being alone in the vehicle, and a sense of complacency about the reliability of the automation. All of these factors combined to produce the collision. 
Figure B.3: Fault Tree Analysis of the Uber collision with a pedestrian

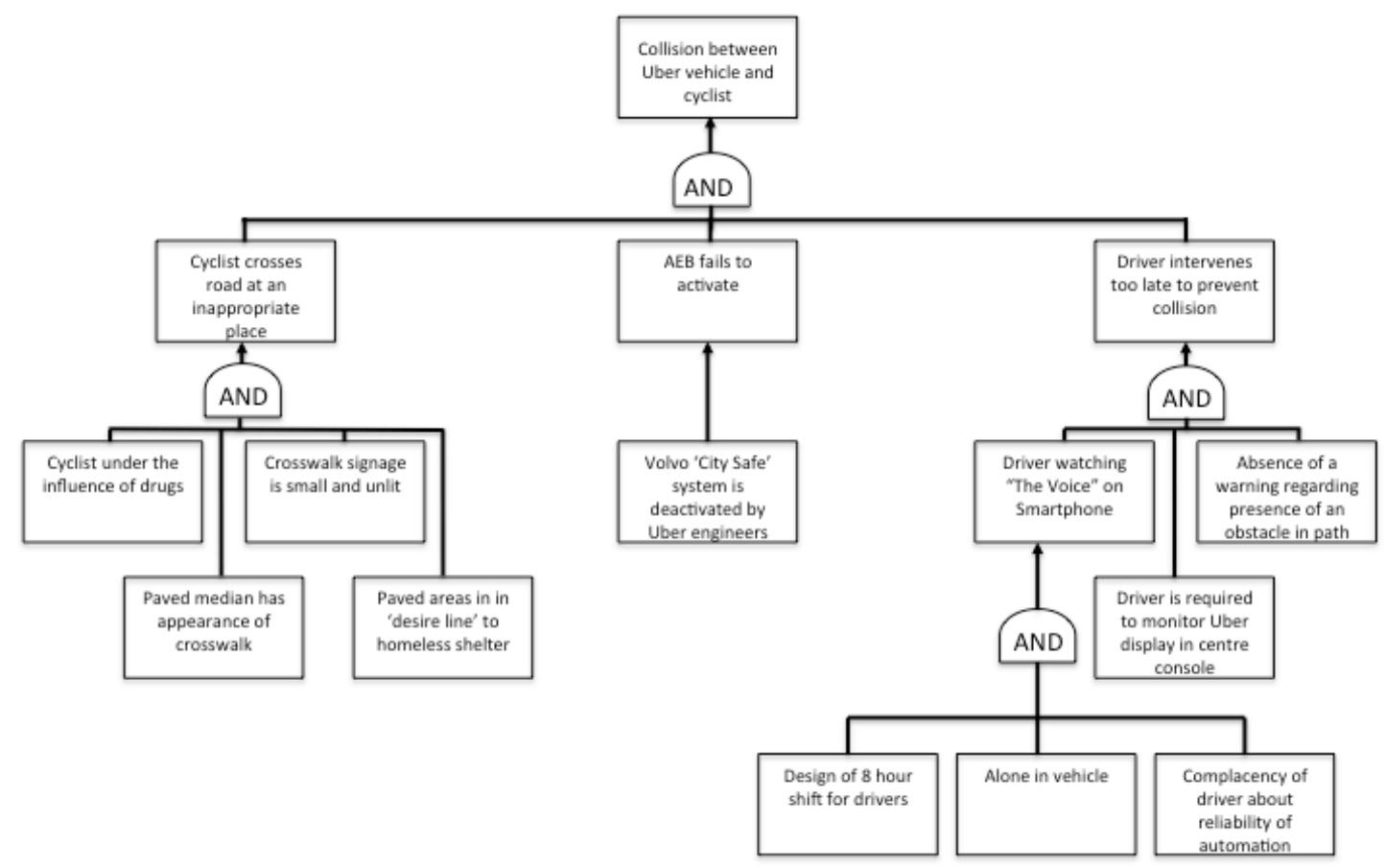

FRAM (Functional Resonance Analysis Method) represents the function involved in the collision between the Uber vehicle and the pedestrian. Those functions associated with performance variability (i.e. functions can become off-nominal and abnormal) are represented with a red outline in Figure B.4. All other functions performed normally.

The functions in the bottom left of Figure B.4 relate to the pedestrian who, apparently, did not read the pedestrian crossing sign, find a safe place to cross or check the road for traffic. The functions on the right-hand side of Figure B.4 relate to the Uber automation and the driver. The Uber automation had some initial difficulty in classifying the pedestrian, but did not warn the driver. The Uber automation was also unable to control the vehicle in an emergency because the Uber engineers had disengaged the AEB. Finally, the driver did not monitor the driving environment adequately nor control the vehicle before the collision.

So, as can be seen in Figure B.4, 7/13 functions did not behave in the manner expected for safe operation of the system. In terms of FRAM, the performance variability of these functions by the pedestrian, the Uber vehicle and the driver led to the collision. 
Figure B.4: FRAM analysis of the Uber collision with a pedestrian

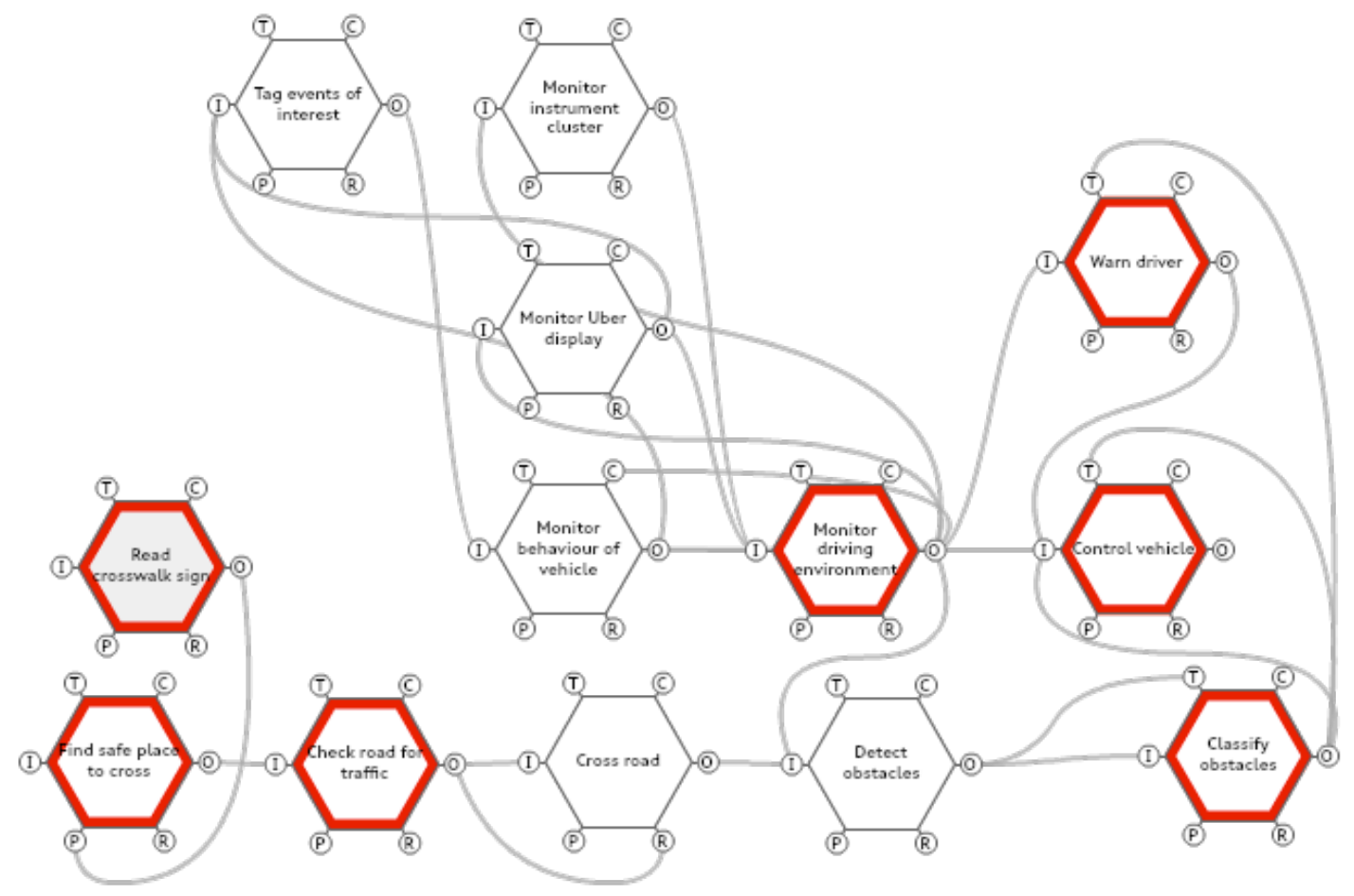

HFACS (Human Factors Analysis and Classification Scheme) is used to classify the system failure for the Uber collision with a pedestrian in Table B.1. The four system categories are in the left-hand column, subcategories in the middle column, and failures in the right-hand column.

As shown in Table B.1, under 'organisational influences', the Uber organisation had failed to identify risky behaviours (by the engineers and drivers) in the testing programme (such as disabling safety systems and attending to smartphones instead of the road). A comprehensive human and technical risk assessment is required to identify these factors.

Under 'supervisory factors', the prescribed tasks were a high workload for one driver: monitor the road environment for hazards, monitor the instrument cluster, monitor the Uber vehicle for problems, monitor the Uber console for anomalies, note any issues on the Uber tablet, and be prepared to take manual control of the vehicle in case of problems. Despite this overtasking, there was also a failure to identify and ameliorate risk behaviours of drivers in the plans for the testing programme. This analysis could have alerted Uber to the issue of the driver not attending to the road environment.

Four 'preconditions for unsafe acts' were noted. For the physical environment, the paved area in the central reservation looked like a crossing, which is probably why the no-crossing signage was posted. Additionally, the signage was small and unlit and would be difficult to see at night. As for the technical environment preconditions, the Uber engineers had disabled the Volvo City Safety system and with it the AEB. The Uber automation also had problems classifying the pedestrian. Regarding the adverse psychological state, the 
pedestrian was intoxicated; and for physical/mental limitations, the driver had many competing tasks.

Finally, the 'unsafe acts' comprised decision errors (the pedestrian not using the proper pedestrian crossing), perceptual errors (driver not having appropriate awareness of the road environment) and routine violations (driver allegedly watching The Voice on a smartphone).

Table B.1: HFACS analysis of the Uber collision with a pedestrian

\begin{tabular}{|c|c|c|}
\hline Category & Subcategory & Failure \\
\hline $\begin{array}{l}\text { Organisational } \\
\text { influences }\end{array}$ & Organisational process & $\begin{array}{l}\text { Failure to identify risky behaviours } \\
\text { in testing programme }\end{array}$ \\
\hline \multirow{2}{*}{$\begin{array}{l}\text { Supervisory } \\
\text { factors }\end{array}$} & Inadequate supervision & Overtasking of driver \\
\hline & $\begin{array}{l}\text { Failure to correct a } \\
\text { problem }\end{array}$ & $\begin{array}{l}\text { Failure to identify risky behaviours } \\
\text { of drivers in plans for testing } \\
\text { programme }\end{array}$ \\
\hline \multirow[t]{4}{*}{$\begin{array}{l}\text { Preconditions } \\
\text { for unsafe acts }\end{array}$} & Physical environment & $\begin{array}{l}\text { Design of paved area (looks like } \\
\text { crossing) } \\
\text { Failure to light signage }\end{array}$ \\
\hline & $\begin{array}{l}\text { Technological } \\
\text { environment }\end{array}$ & $\begin{array}{l}\text { Disabling of Volvo City Safety } \\
\text { Misclassification of obstacle }\end{array}$ \\
\hline & $\begin{array}{l}\text { Adverse physiological } \\
\text { state }\end{array}$ & Pedestrian intoxication \\
\hline & $\begin{array}{l}\text { Physical/mental } \\
\text { limitations }\end{array}$ & Conflicting driver tasks \\
\hline \multirow[t]{3}{*}{ Unsafe acts } & Decision errors & $\begin{array}{l}\text { Failure of pedestrian to use } \\
\text { pedestrian crossing }\end{array}$ \\
\hline & Perceptual errors & $\begin{array}{l}\text { Driver losing situation awareness } \\
\text { of road environment }\end{array}$ \\
\hline & Routine violations & $\begin{array}{l}\text { Driver allegedly watching The } \\
\text { Voice on smartphone }\end{array}$ \\
\hline
\end{tabular}

STAMP-CAST (Systems-Theoretic Accident Model and Processes - Causal Analysis using Systems Theory) shows the hierarchy of organisations in Figure B.5, from the international context at the highest level to the operating environment and operating process at the lowest level. At each level, the key hazards were identified in terms of control flaws, such as inadequate 
enforcement of constraints, inadequate execution of control actions and inadequate or missing feedback.

At the level of international context, Volvo had allowed the Uber engineers to disable the City Safety system and there were no international standards for safe vehicle automation. The federal and state government did not have regulations for risk assessment and safe testing of automated vehicles. At the level of industry associations, there are no SAE (Society of Automotive Engineers) standards for testing vehicle automation on the road. Local government had developed a paved central reservation that looked like a pedestrian crossing, and installed a small, unlit sign to say that it was not a pedestrian crossing. Uber's training did not guard against drivers engaging with smartphones while driving, or against its engineers disabling the Volvo City Safety system (thereby removing the safety net of the AEB). In the operating environment, the pedestrian did not obey the pedestrian crossing sign (probably as a consequence of the combination of the walkway being on the preferred path, the low visibility at night and the small signage). In the operating process, there was no warning to the driver about the presence of a pedestrian, and the AEB did not activate (because it had been disabled). There was initial misclassification of the pedestrian by the Uber systems, showing an interaction between the operating process and environment.

What the STAMP-CAST analysis shows is failures at all levels in the systems, to do mainly with control rather than feedback.

\section{Figure B.5: STAMP-CAST analysis of the Uber collision with a pedestrian}

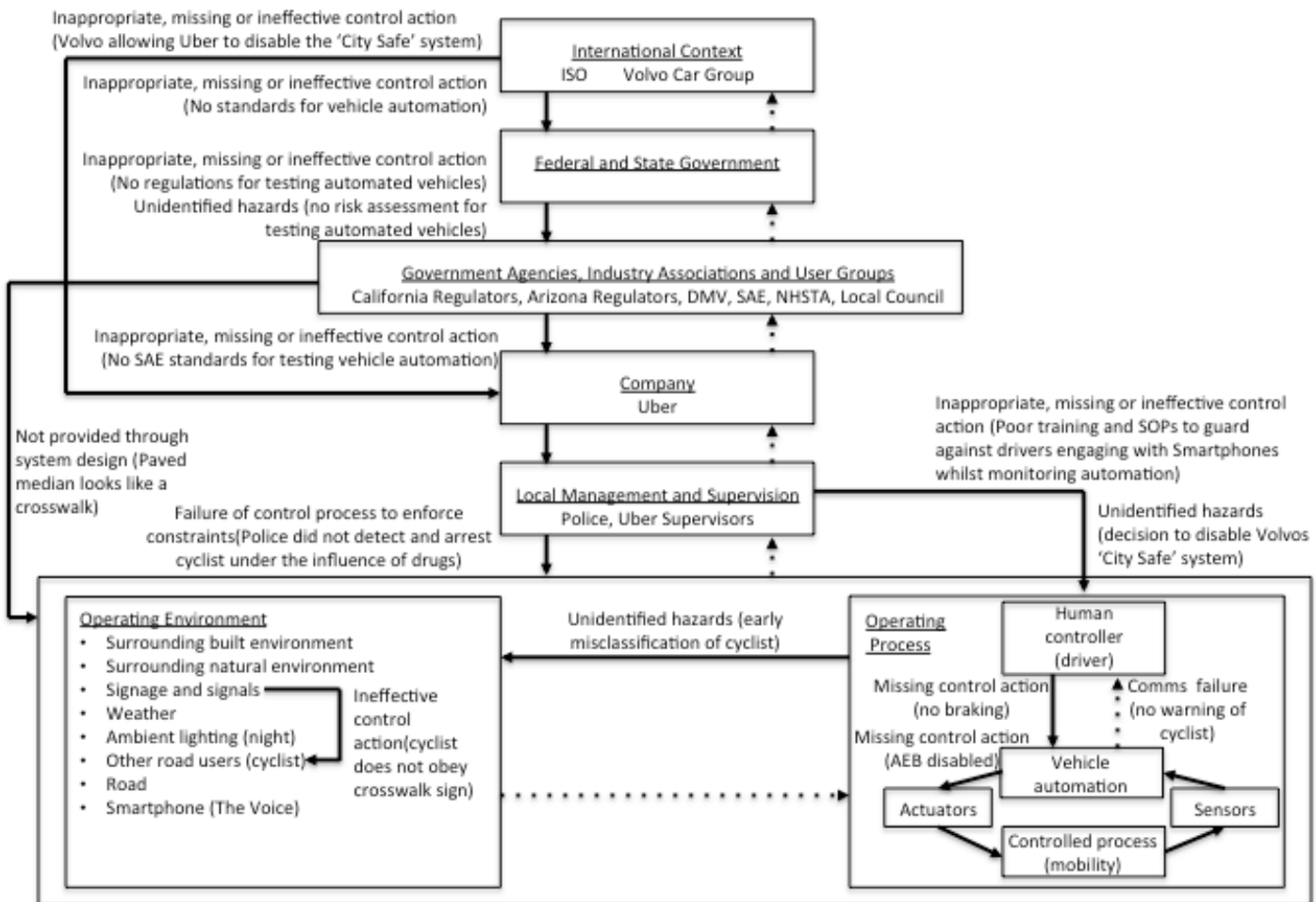


STEP (Sequential Timed Event Plotting) shows the timed sequence of events leading up to the collision between the Uber vehicle and the pedestrian in Figure B.6. (The red box highlights the collision)

The sequence begins with the driver taking the Volvo out of the garage at 21.14. Then it is alleged that The Voice is streamed on the driver's phone. The driver engaged automation mode at 21.39 and the vehicle proceeds around a preset route. A police report states that the driver looks down 204 times over the course of 11.8 miles. It is calculated that the driver's eyes were off of the road for $6 \mathrm{~min} 47 \mathrm{sec}$ in the period immediately prior to the collision.

At some point during this time, a pedestrian wheeling a bicycle decides to cross the road. The road is dark and unlit at the central reservation when the pedestrian crosses. There is a small, unlit sign stating that the pedestrian crossing is further up the road. The Uber system detects an obstacle in its path and initially misclassifies it (originally as unclassified, then as a car, then as a bicycle). Then the system called for the AEB to be activated, but the Uber engineers already disabled it.

About half a second before the collision, the driver looks up and spots the pedestrian. The driver attempts to steer the vehicle away from the pedestrian but they are struck at $39 \mathrm{mph}$. Approximately one second after the collision, the driver applied the brakes. When the vehicle stops the driver calls 911 to report the collision, and then switches off the phone.

Figure B.6: STEP analysis of the Uber collision with a pedestrian

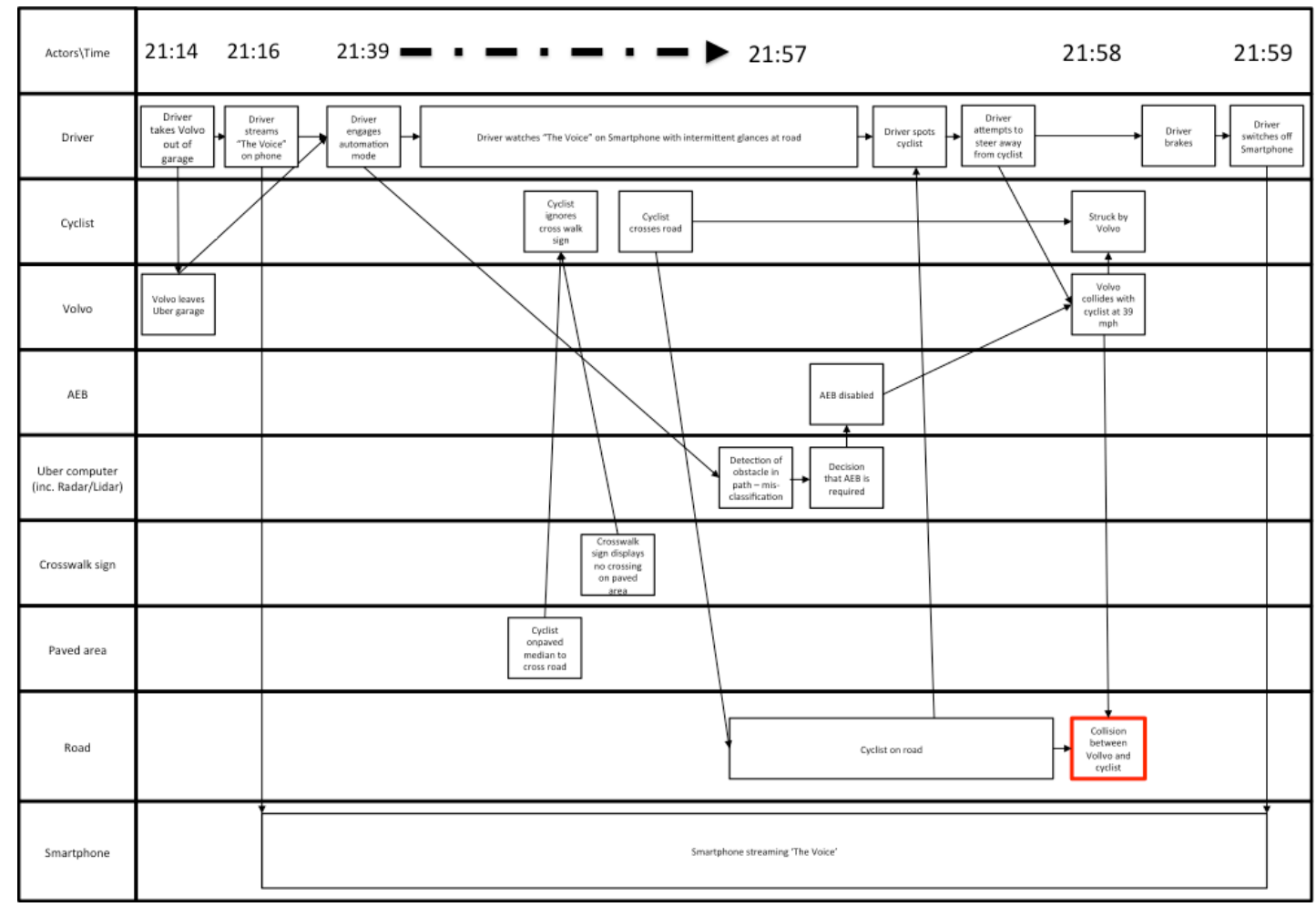

